\title{
CABLE LINKS AND L-SPACE SURGERIES
}

\author{
EUGENE GORSKY AND JENNIFER HOM
}

\begin{abstract}
An L-space link is a link in $S^{3}$ on which all sufficiently large integral surgeries are L-spaces. We prove that for $m, n$ relatively prime, the $r$-component cable link $K_{r m, r n}$ is an L-space link if and only if $K$ is an L-space knot and $n / m \geq 2 g(K)-1$. We also compute $\mathrm{HFL}^{-}$and $\widehat{\mathrm{HFL}}$ of an L-space cable link in terms of its Alexander polynomial. As an application, we confirm a conjecture of Licata [Lic12] regarding the structure of $\widehat{\mathrm{HFL}}$ for $(n, n)$ torus links.
\end{abstract}

\section{INTRODUCTION}

Heegaard Floer homology is a package of 3-manifold invariants defined by Ozsváth and Szabó [OS04a, OS04b]. In its simplest form, it associates to a closed 3-manifold $Y$ a graded vector space $\widehat{\mathrm{HF}}(Y)$. For a rational homology sphere $Y$, they show that

$$
\operatorname{dim} \widehat{\mathrm{HF}}(Y) \geq\left|H_{1}(Y ; \mathbb{Z})\right| \text {. }
$$

If equality is achieved, then $Y$ is called an $L$-space.

A knot $K \subset S^{3}$ is an $L$-space knot if $K$ admits a positive L-space surgery. Let $S_{p / q}^{3}(K)$ denote $p / q$ Dehn surgery along $K$. If $K$ is an L-space knot, then $S_{p / q}^{3}(K)$ is an L-space for all $p / q \geq 2 g(K)-1$, where $g(K)$ denotes the Seifert genus of $K$ [OS11, Corollary 1.4]. A link $L \subset S^{3}$ is an $L$-space link if all sufficiently large integral surgeries on $L$ are L-spaces. In contrast to the knot case, if $L$ admits a positive L-space surgery, it does not necessarily follow that all sufficiently large surgeries are also L-spaces; see [Liu14, Example 2.3].

For relatively prime integers $m$ and $n$, let $K_{m, n}$ denote the $(m, n)$ cable of $K$, where $m$ denotes the longitudinal winding. Without loss of generality, we will assume that $m>0$. Work of Hedden [Hed09] ("if" direction) and the second author [Hom11] ("only if" direction) completely classifies L-space cable knots.

Theorem 1 ([Hed09, Hom11]). Let $K$ be a knot in $S^{3}, m>1$ and $\operatorname{gcd}(m, n)=1$. The cable knot $K_{m, n}$ is an L-space knot if and only if $K$ is an L-space knot and $n / m>2 g(K)-1$.

Remark 1.1. Note that when $m=1$, we have that $K_{1, n}=K$ for all $n$.

We generalize this theorem to cable links with many components. Throughout the paper, we assume that each component of a cable link is oriented in the same direction.

Theorem 2. Let $K$ be a knot in $S^{3}$ and $\operatorname{gcd}(m, n)=1$. The $r$-component cable link $K_{r m, r n}$ is an $L$-space link if and only if $K$ is an L-space knot and $n / m \geq 2 g(K)-1$.

In [OS05], Ozsváth and Szabó show that if $K$ is an L-space knot, then $\widehat{\operatorname{HFK}}(K)$ is completely determined by $\Delta_{K}(t)$, the Alexander polynomial of $K$. Consequently, the Alexander polynomials of L-space knots are quite constrained (the non-zero coefficients are all \pm 1 and alternate in sign)

The first author was partially supported by RFBR grant 13-01-00755 and NSF grant DMS-1403560.

The second author was partially supported by NSF grant DMS-1307879. 
and the rank of $\widehat{\mathrm{HFK}}(K)$ is at most one in each Alexander grading. In [Liu14, Theorem 1.15], Liu generalizes this result to give bounds on the rank of $\mathrm{HFL}^{-}(L)$ in each Alexander multi-grading and on the coefficients of the multi-variable Alexander polynomial of an L-space link $L$ in terms of the number of components of $L$. For L-space cable links, we have the following stronger result.

Definition 1.2. Define the $\mathbb{Z}$-valued functions $\mathbf{h}(k)$ and $\beta(k)$ by the equations:

$$
\sum_{k} \mathbf{h}(k) t^{k}=\frac{t^{-1} \Delta_{m, n}(t)\left(t^{m n r / 2}-t^{-m n r / 2}\right)}{\left(1-t^{-1}\right)^{2}\left(t^{m n / 2}-t^{-m n / 2}\right)}, \quad \beta(k)=\mathbf{h}(k-1)-\mathbf{h}(k)-1,
$$

where $\Delta_{m, n}(t)$ is the Alexander polynomial of the cable knot $K_{m, n}$.

Throughout, we work with $\mathbb{F}=\mathbb{Z} / 2 \mathbb{Z}$ coefficients. The following theorem gives a complete description of the homology groups $\widehat{\mathrm{HFL}}$ for cable links with $n / m>2 g(K)-1$.

Theorem 3. Let $K_{r m, r n}$ be a cable link with $n / m>2 g(K)-1$.

(a) If $\beta(k)+\beta(k+1) \leq r-2$ then:

$$
\widehat{\operatorname{HFL}}\left(K_{r m, r n}, k, \ldots, k\right) \simeq \bigoplus_{i=0}^{\beta(k)}\left(\begin{array}{c}
r-1 \\
i
\end{array}\right) \mathbb{F}_{-2 \mathbf{h}(k)-i} \oplus \bigoplus_{i=0}^{\beta(k+1)}\left(\begin{array}{c}
r-1 \\
i
\end{array}\right) \mathbb{F}_{-2 \mathbf{h}(k)+2-r+i}
$$

(b) If $\beta(k)+\beta(k+1) \geq r-2$ then:

$$
\widehat{\mathrm{HFL}}\left(K_{r m, r n}, k, \ldots, k\right) \simeq \bigoplus_{i=0}^{r-2-\beta(k+1)}\left(\begin{array}{c}
r-1 \\
i
\end{array}\right) \mathbb{F}_{-2 \mathbf{h}(k)-i} \oplus \bigoplus_{i=0}^{r-2-\beta(k)}\left(\begin{array}{c}
r-1 \\
i
\end{array}\right) \mathbb{F}_{-2 \mathbf{h}(k)+2-r+i}
$$

(c) If $v$ has $j$ coordinates equal to $k-1$ and $r-j$ coordinates equal to $k$ for some $k$ and $1 \leq j \leq r-1$, then:

$$
\widehat{\operatorname{HFL}}\left(K_{r m, r n},(k-1)^{j}, k^{r-j}\right) \simeq\left(\begin{array}{c}
r-2 \\
\beta(k)
\end{array}\right) \mathbb{F}_{-2 \mathbf{h}(k)-\beta(k)-j} .
$$

(d) For all other Alexander gradings the groups $\widehat{\mathrm{HFL}}$ vanish.

We prove the parts of this theorem as separate Theorems 4.22, 4.24 and 4.25 . We compute $\widehat{\mathrm{HFL}}$ explicitly for several examples in Section 5. In particular, we use Theorem 3 to confirm a conjecture of Joan Licata [Lic12, Conjecture 1] concerning $\widehat{\mathrm{HFL}}$ for $(n, n)$ torus links.

Theorem 4. Suppose that $0 \leq s \leq \frac{n-1}{2}$. Then

$$
\widehat{\operatorname{HFL}}\left(T(n, n), \frac{n-1}{2}-s, \ldots, \frac{n-1}{2}-s\right)=\bigoplus_{i=0}^{s}\left(\begin{array}{c}
n-1 \\
i
\end{array}\right) \mathbb{F}_{\left(-s^{2}-s-i\right)} \oplus \bigoplus_{i=0}^{s-1}\left(\begin{array}{c}
n-1 \\
i
\end{array}\right) \mathbb{F}_{\left(-s^{2}-s-n+2+i\right)} .
$$

Combined with [Lic12, Theorem 2], this completes the description of $\widehat{\operatorname{HFL}}(T(n, n))$.

The following theorem describes the homology groups $\mathrm{HFL}^{-}$for cable links with $n / m>2 g(K)-1$.

Theorem 5. Let $K$ be an L-space knot and $n / m>2 g(K)-1$. Consider an Alexander grading $v=\left(v_{1}, \ldots, v_{n}\right)$. Suppose that among the coordinates $v_{i}$ exactly $\lambda$ are equal to $k$ and all other coordinates are less than $k$. Let $|v|=v_{1}+\ldots+v_{n}$. Then the Heegaard-Floer homology group $\operatorname{HFL}^{-}\left(K_{r m, r n}, v\right)$ can be described as follows:

(a) If $\beta(k)<r-\lambda$ then $\operatorname{HFL}^{-}\left(K_{r m, r n}, v\right)=0$. 
(b) If $\beta(k) \geq r-\lambda$ then

$$
\operatorname{HFL}^{-}\left(K_{r m, r n}, v\right) \simeq\left(\mathbb{F}_{(0)} \oplus \mathbb{F}_{(-1)}\right)^{r-\lambda} \otimes \bigoplus_{i=0}^{\beta(k)-r+\lambda}\left(\begin{array}{c}
\lambda-1 \\
i
\end{array}\right) \mathbb{F}_{(-2 h(v)-i)},
$$

where $h(v)=\mathbf{h}(k)+k r-|v|$.

We prove this theorem in Section 4.2. The structure of the homology for $n / m=2 g(K)-1$ (which is possible only if $m=1$ ) is more subtle and is described in Theorem 4.26.

Finally, we describe $\mathrm{HFL}^{-}$as an $\mathbb{F}\left[U_{1}, \ldots, U_{r}\right]$-module. We define a collection of $\mathbb{F}\left[U_{1}, \ldots, U_{r}\right]-$ modules $M_{\beta}$ for $0 \leq \beta \leq r-2, M_{r-1, k}$ for $k \geq 0$ and $M_{r-1, \infty}$. These modules can be defined combinatorially and do not depend on a link.

Theorem 6. Let $R=\mathbb{F}\left[U_{1}, \ldots, U_{r}\right]$ and suppose that $n / m>2 g(K)-1$. There exists a finite collection of diagonal lattice points $\mathbf{a}_{i}=\left(a_{i}, \ldots, a_{i}\right)$ (determined by $m, n$ and the Alexander polynomial of $K$ ) such that $\mathrm{HFL}^{-}$admits the following direct sum decomposition:

$$
\operatorname{HFL}^{-}\left(K_{r m, r n}\right)=\bigoplus_{i} R \cdot \operatorname{HFL}^{-}\left(K_{r m, r n}, \mathbf{a}_{i}\right) .
$$

Furthermore, for $\beta\left(a_{i}\right) \leq r-2$ one has $R \cdot \operatorname{HFL}^{-}\left(K_{r m, r n}, \mathbf{a}_{i}\right) \simeq M_{\beta\left(a_{i}\right)}$, and for $\beta\left(a_{i}\right)=r-1$ one has either $R \cdot \operatorname{HFL}^{-}\left(K_{r m, r n}, \mathbf{a}_{i}\right) \simeq M_{r-1, k}$ for some $k$ or $R \cdot \operatorname{HFL}^{-}\left(K_{r m, r n}, \mathbf{a}_{i}\right) \simeq M_{r-1, \infty}$.

We compute $\mathrm{HFL}^{-}$explicitly for several examples in Section 5.

\section{ACKNOWLEDGMENTS}

We are grateful to Jonathan Hanselman, Matt Hedden, Yajing Liu, Joan Licata, and András Némethi for useful discussions.

\section{DEHN SURGERY AND CABLE LINKS}

In this section, we prove Theorem 2. We begin with a result about Dehn surgery on cable links (cf. [Hei74]).

Proposition 2.1. The manifold obtained by $\left(m n, p_{2}, \ldots, p_{r}\right)$-surgery on the $r$-component link $K_{r m, r n}$ is homeomorphic to $S_{n / m}^{3}(K) \# L(m, n) \# L\left(p_{2}-m n, 1\right) \# \ldots \# L\left(p_{r}-m n, 1\right)$.

Proof. Recall (see, for example, [Hed09, Section 2.4]) that $m n$-surgery on $K_{m, n}$ gives the manifold $S_{n / m}^{3}(K) \# L(m, n)$. Viewing $K_{m, n}$ as the image of $T_{m, n}$ on $\partial N(K)$, we have that the reducing sphere is given by the annulus $\partial N(K) \backslash N\left(T_{m, n}\right)$ union two parallel copies of the meridional disk of the surgery solid torus; we obtain a sphere since the surgery slope coincides with the surface framing.

The link $K_{r m, r n}$ consists of $r$ parallel copies of $K_{m, n}$ on $\partial N(K)$. Label these $r$ copies $K_{m, n}^{1}$ through $K_{m, n}^{r}$. We perform $m n$-surgery on $K_{m, n}^{1}$ and consider the image $\widetilde{K}_{m, n}^{i}$ of $K_{m, n}^{i}, 2 \leq i \leq r$, in $S_{n / m}^{3}(K) \# L(m, n)$. Each $\widetilde{K}_{m, n}^{i}$ lies on $\partial N(K) \backslash N\left(T_{m, n}\right)$ and thus on the reducing sphere. In particular, each $\widetilde{K}_{m, n}^{i}$ bounds a disk $D_{i}^{2}$ in $S_{n / m}^{3}(K) \# L(m, n)$ such that the collection $\left\{D_{2}^{2}, \ldots, D_{r}^{2}\right\}$ is disjoint. It follows that performing surgery on $\bigcup_{i=2}^{r} \widetilde{K}_{m, n}^{i}$ yields $r-1$ lens space summands. To see which lens spaces we obtain, note that the $m n$-framed longitude on $K_{m, n}^{i} \subset S^{3}$ coincides with

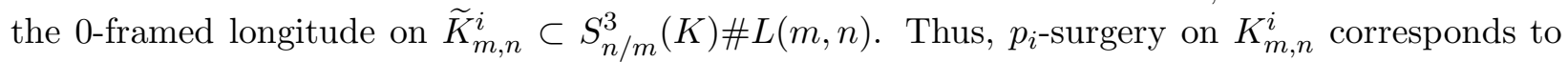
$\left(p_{i}-m n\right)$-surgery on $\widetilde{K}_{m, n}^{i}$, and the result follows. 
Let us recall that the linking number between each two components of $K_{r m, r n}$ equals $l:=m n$. It is well-known that the cardinality of $H_{1}$ of the manifold obtained by $\left(p_{1}, p_{2}, \ldots, p_{r}\right)$-surgery on $K_{r m, r n}$ equals $\left|\operatorname{det} \Lambda\left(p_{1}, \ldots, p_{r}\right)\right|$, where

$$
\Lambda_{i j}= \begin{cases}p_{i}, & \text { if } i=j, \\ l, & \text { if } i \neq j .\end{cases}
$$

This cardinality can be computed using the following result.

Proposition 2.2. One has the following identity:

$$
\operatorname{det} \Lambda\left(p_{1}, \ldots, p_{r}\right)=\left(p_{1}-l\right) \cdots\left(p_{r}-l\right)+l \sum_{i=1}^{r}\left(p_{1}-l\right) \cdots\left(\widehat{p_{i}-l}\right) \cdots\left(p_{r}-l\right) .
$$

Proof. One can easily check that $\operatorname{det} \Lambda\left(l, p_{2}, \ldots, p_{r}\right)=l\left(p_{2}-l\right) \cdots\left(p_{r}-l\right)$. The expansion of the determinant in the first row yields a recursion relation

$$
\begin{gathered}
\operatorname{det} \Lambda\left(p_{1}, \ldots, p_{r}\right)=\operatorname{det} \Lambda\left(l, p_{2} \ldots, p_{r}\right)+\left(p_{1}-l\right) \operatorname{det} \Lambda\left(p_{2}, \ldots, p_{r}\right)= \\
=l\left(p_{2}-l\right) \cdots\left(p_{r}-l\right)+\left(p_{1}-l\right) \operatorname{det} \Lambda\left(p_{2}, \ldots, p_{r}\right) .
\end{gathered}
$$

Now (2.1) follows by induction in $r$.

Corollary 2.3. If $p_{i} \geq l$ for all $i$ then $\operatorname{det} \Lambda\left(p_{1}, \ldots, p_{r}\right) \geq 0$.

In order to prove Theorem 2, we will need the following:

Theorem 2.4 ([Liu14, Proposition 1.11]). A link $L$ is an $L$-space link if and only if there exists a surgery framing $\Lambda\left(p_{1}, \ldots, p_{r}\right)$, such that for all sublinks $L^{\prime} \subseteq L, \operatorname{det}\left(\left.\Lambda\left(p_{1}, \ldots, p_{r}\right)\right|_{L^{\prime}}\right)>0$ and $S_{\left.\Lambda\right|_{L^{\prime}}}^{3}\left(L^{\prime}\right)$ is an $L$-space.

We will also need the following proposition, which we prove in Subsection 2.1 below.

Proposition 2.5. Let $K$ be an $L$-space knot and $p_{i}>0, i=1, \ldots, r$. If $n<2 g(K)-1$, then the manifold obtained by $\left(p_{1}, \ldots, p_{r}\right)$-surgery on the $r$-component link $K_{r, r n}$ is not an L-space.

Proof of Theorem 2. If $K_{r m, r n}$ is an L-space link, then by [Liu14, Lemma 1.10] all its components are L-space knots. On the other hand, its components are isotopic to $K_{m, n}$. Thus, if $m>1$, then by Theorem $1, K$ is an L-space knot and $n / m>2 g(K)-1$. If $m=1$, then $K$ must be an L-space knot and by Proposition 2.5, $n \geq 2 g(K)-1$.

Conversely, suppose that $K$ is an L-space knot and $n / m \geq 2 g(K)-1$, i.e., $K_{m, n}$ is an Lspace knot. Let us prove by induction on $r$ that $\left(p_{1}, \ldots, p_{r}\right)$-surgery on $K_{r m, r n}$ is an L-space if $p_{i}>l$ for all $i$. For $r=1$ it is clear. By Proposition 2.1, the link $K_{r m, r n}$ admits an L-space surgery with parameters $l, p_{2}, \ldots, p_{r}$. Let us apply Theorem 2.4. Indeed, by Corollary 2.3, one has $\operatorname{det}\left(\left.\Lambda\left(l, p_{2} \ldots, p_{r}\right)\right|_{L^{\prime}}\right)>0$ and by the induction assumption $S_{\left.\Lambda\left(l, p_{2} \ldots, p_{r}\right)\right|_{L^{\prime}}}^{3}\left(L^{\prime}\right)$ is an L-space for all sublinks $L^{\prime}$. By [Liu14, Lemma 2.5], $\left(p_{1}, \ldots, p_{r}\right)$-surgery on $K_{r m, r n}$ is also an L-space for all $p_{1}>l$. Therefore $K_{r m, r n}$ is an L-space link.

2.1. Proof of Proposition 2.5. We will prove Proposition 2.5 using Lipshitz-Ozsváth-Thurston's bordered Floer homology [LOT08], specifically Hanselman-Watson's [HW15] loop calculus. That is, we will decompose the result of surgery on $K_{r, r n}$ into two pieces, one that is surgery on a torus link in the solid torus and the other the knot complement, and then apply a gluing result of Hanselman-Watson to conclude that the result of this surgery along $K_{r, r n}$ is not an L-space. The following was described to us by Jonathan Hanselman. 
Let $Y_{1}$ denote the Seifert fibered space obtained by performing $\left(p_{1}, \ldots, p_{r}\right)$-surgery on the $r$ component $(r, 0)$-torus link in the solid torus. Consider the bordered manifold $\left(Y_{1}, \alpha_{1}, \beta_{1}\right)$, where $\alpha_{1}$ is the fiber slope and $\beta_{1}$ lies in the base orbifold; that is, $\alpha_{1}$ is the longitude and $\beta_{1}$ the meridian of the original solid torus. Let $\left(Y_{2}, \alpha_{2}, \beta_{2}\right)$ be the $n$-framed complement of $K$; that is, $Y_{2}=S^{3} \backslash N(K)$, $\alpha_{2}$ is an $n$-framed longitude, and $\beta_{2}$ is a meridian. Let $\left(Y_{1}, \alpha_{1}, \beta_{1}\right) \cup\left(Y_{2}, \alpha_{2}, \beta_{2}\right)$ denote the result of gluing $Y_{1}$ to $Y_{2}$ by identifying $\alpha_{1}$ with $\alpha_{2}$ and $\beta_{1}$ with $\beta_{2}$. Note that $\left(Y_{1}, \alpha_{1}, \beta_{1}\right) \cup\left(Y_{2}, \alpha_{2}, \beta_{2}\right)$ is homeomorphic to $\left(p_{1}, \ldots, p_{r}\right)$-surgery along $K_{r, r n}$. We identify the slope $p \alpha_{i}+q \beta_{i}$ on $\partial Y_{i}$ with the (extended) rational number $\frac{p}{q} \in \mathbb{Q} \cup\left\{\frac{1}{0}\right\}$.

The following lemma gives a description of $\widehat{\mathrm{CFD}}\left(Y_{1}, \alpha_{1}, \beta_{1}\right)$ in terms of the standard notation defined in [HW15, Section 3.2].

Lemma 2.6. The invariant $\widehat{\mathrm{CFD}}\left(Y_{1}, \alpha_{1}, \beta_{1}\right)$ can be written in standard notation as a product of $d_{k_{i}}$ where

(1) $k_{i} \leq 0$ for all $i$,

(2) $k_{i}=0$ for at least one $i$,

(3) $k_{i}=-r$ for exactly one $i$.

Proof. The computation is similar to the example in [HW15, Section 6.5]. A plumbing tree $\Gamma$ for $Y_{1}$ is given in Figure 1. We first consider the plumbing tree $\Gamma_{i}$ in Figure 2(a). We will build $\Gamma$ by merging the $\Gamma_{i}, i=1, \ldots, r$.

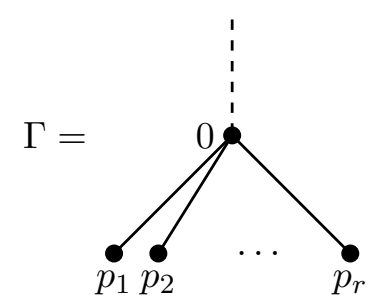

Figure 1. The plumbing tree $\Gamma$.

We proceed as in [HW15, Section 6.5]. Start with a loop $\left(d_{0}\right)$ representing the tree $\Gamma_{0}$ in Figure 2(b). We have that $\Gamma_{i}=\mathcal{E}\left(\mathcal{T}^{p}\left(\Gamma_{0}\right)\right)$ so by [HW15, Sections 3.3 and 6.3]:

$$
\begin{aligned}
& \widehat{\mathrm{CFD}}\left(\Gamma_{i}\right)=\mathrm{E}\left(\mathrm{T}^{p_{i}}\left(\left(d_{0}\right)\right)\right) \\
& =\mathrm{E}\left(\left(d_{p_{i}}\right)\right) \\
& =\left(d_{-p_{i}}^{*}\right) \\
& \sim(d_{-1} \underbrace{d_{0} \ldots d_{0}}_{p_{i}}) \text {. }
\end{aligned}
$$
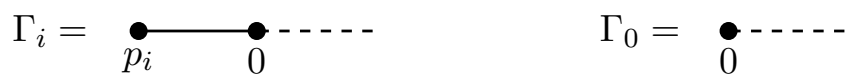

(a) (b)

Figure 2. Left, the plumbing tree $\Gamma_{i}$. Right, the plumbing tree $\Gamma_{0}$. 
We then have that $\Gamma=\mathcal{M}\left(\Gamma_{2}, \mathcal{M}\left(\Gamma_{2}, \ldots, \mathcal{M}\left(\Gamma_{p_{r-1}}, \Gamma_{p_{r}}\right)\right)\right)$. By [HW15, Proposition 6.4], we have that $\widehat{\mathrm{CFD}}(\Gamma)$ is a represented by a product of $d_{k_{i}}$ where $k_{i} \leq 0$ for all $i$ and $k_{i}=0$ for at least one $i$ since each $p_{i}>0$. Moreover, $d_{-r}$ appears exactly once in the product, since we performed $r-1$ merges. This completes the proof of the lemma.

Lemma 2.7. The slope 1 is not a strict L-space slope on $\left(Y_{1}, \alpha_{1}, \beta_{1}\right)$.

Proof. We will apply a positive Dehn twist to $\left(Y_{1}, \alpha_{1}, \beta_{1}\right)$ to obtain $\left(Y_{1}, \alpha_{1}, \beta_{1}+\alpha_{1}\right)$. We will show that 0 is not a strict L-space slope on $\left(Y_{1}, \alpha_{1}, \beta_{1}+\alpha_{1}\right)$, and hence 1 is not a strict L-space slope on $\left(Y_{1}, \alpha_{1}, \beta_{1}\right)$.

By [HW15, Proposition 6.1], we have that $\widehat{\mathrm{CFD}}\left(Y_{1}, \alpha_{1}, \beta_{1}+\alpha_{1}\right)$ can be obtained by applying $\mathrm{T}$ to a loop representative of $\widehat{\operatorname{CFD}}\left(Y_{1}, \alpha_{1}, \beta_{1}\right)$. Since $\mathrm{T}\left(d_{k}\right)=d_{k+1}$, it follows from Lemma 2.6 that $\widehat{\mathrm{CFD}}\left(Y_{1}, \alpha_{1}, \beta_{1}+\alpha_{1}\right)$ can be written in standard notation as a product of $d_{k_{i}}$ with $k_{i} \leq 1$ for all $i$, $k_{i}=1$ for at least one $i$, and $k_{i}=1-r$ for exactly one $i$.

We claim that if a loop $\ell$ contains both positive and negative $d_{k}$ segments (i.e., both $d_{i}, i>0$ and $\left.d_{j}, j<0\right)$, then in dual notation $\ell$ contains at least one $a_{i}^{*}$ or $b_{j}^{*}$ segment. Indeed, suppose by contradiction that $\boldsymbol{\ell}$ has no $a_{i}^{*}$ or $b_{j}^{*}$. Then $\boldsymbol{\ell}$ consists of only $d_{i}^{*}$ segments, $i \in \mathbb{Z}$. It is straightforward to see (for example, by considering the segments as drawn in [HW15, Figure 1]) that one cannot obtain a loop containing both positive and negative $d_{k}$ segments from $d_{i}^{*}$ segments, $i \in \mathbb{Z}$. This completes the proof of the claim.

Furthermore, note that $\widehat{\mathrm{CFD}}\left(Y_{1}, \alpha_{1}, \beta_{1}+\alpha_{1}\right)$ consists of simple loops (see Definition 4.19 of [HW15]). Then by [HW15, Proposition 4.24], in dual notation $\ell$ has no $a_{k}^{*}$ or $b_{k}^{*}$ segments for $k<0$. It now follows from Proposition 4.18 of [HW15] that 0 is not a strict L-space slope for $\widehat{\mathrm{CFD}}\left(Y_{1}, \alpha_{1}, \beta_{1}+\alpha_{1}\right)$. Therefore, 1 is not a strict L-space slope on $\left(Y_{1}, \alpha_{1}, \beta_{1}\right)$, as desired.

Remark 2.8. Note that by Proposition 4.18 of [HW15], we have that 0 and $\infty$ are strict L-space slopes on $\left(Y_{1}, \alpha_{1}, \beta_{1}\right)$. Since 1 is not a strict L-space slope, it follows from Corollary 4.5 of [HW15] that the interval of L-space slopes of $\left(Y_{1}, \alpha_{1}, \beta_{1}\right)$ contains the interval $[-\infty, 0]$.

Remark 2.9. An alternative proof of Lemma 2.7 follows from [LS07, Theorem 1.1]. Indeed, by setting $r_{i}=1 / p_{i}$ and $e_{0}=-1$ in Figure 1 of [LS07], we see that $M\left(-1 ; 1 / p_{1}, \ldots, 1 / p_{r}\right)$ is not an L-space, hence neither is $M\left(1 ;-1 / p_{1}, \ldots,-1 / p_{r}\right)$, which is homeomorphic to filling $\left(Y_{1}, \alpha_{1}, \beta_{1}\right)$ along a curve of slope 1 .

Lemma 2.10. Let $K$ be an L-space knot. If $n<2 g(K)-1$, then 1 is not a strict $L$-space slope on the $n$-framed knot complement $\left(Y_{2}, \alpha_{2}, \beta_{2}\right)$.

Proof. Since $K$ is an L-space knot, we have that $S_{K}^{3}(p / q)$ is an L-space exactly when $p / q \geq$ $2 g(K)-1$. Since $\alpha_{2}$ is an $n$-framed longitude, it follows that the interval of strict L-space slopes on $\left(Y_{2}, \alpha_{2}, \beta_{2}\right)$ is $\left(0, \frac{1}{2 g(K)-1-n}\right)$, that is, the reciprocal of the interval $(2 g(K)-1-n, \infty)$.

Proof of Proposition 2.5. The result now follows from [HW15, Theorem 1.3] combined with Lemmas 2.7 and 2.10; the slope 1 is not a strict L-space slope on either $\left(Y_{1}, \alpha_{1}, \beta_{1}\right)$ or $\left(Y_{2}, \alpha_{2}, \beta_{2}\right)$, and so the resulting manifold $\left(Y_{1}, \alpha_{1}, \beta_{1}\right) \cup\left(Y_{2}, \alpha_{2}, \beta_{2}\right)$, which is $\left(p_{1}, \ldots, p_{r}\right)$-surgery on $K_{r, r n}$, is not an L-space.

Remark 2.11. One can use similar methods to provide an alternate proof that $K_{r, r n}$ is an L-space link if $K$ is an L-space knot and $n \geq 2 g(K)-1$. Indeed, if $K$ is an L-space knot, then the interval of strict L-space slopes on the $n$-framed knot complement $\left(Y_{2}, \alpha_{2}, \beta_{2}\right)$ is $\left(0, \frac{1}{2 g(K)-1-n}\right)$ if $n \leq 2 g(K)-1$ and $(0, \infty] \cup\left[-\infty, \frac{1}{2 g(K)-1-n}\right)$ if $n>2 g(K)-1$. Hence if $n \geq 2 g(K)-1$, then the 
interval of strict L-space slopes on $\left(Y_{2}, \alpha_{2}, \beta_{2}\right)$ contains the interval $(0, \infty)$. By Remark 2.8 , we have that the interval of strict L-space slopes on $\left(Y_{1}, \alpha_{1}, \beta_{1}\right)$ contains $[-\infty, 0]$. Therefore, by [HW15, Theorem 1.4], if $n \geq 2 g(K)=1$, then the result of positive surgery (i.e., each surgery coefficient is positive) on $K_{r, r n}$ is an L-space.

\section{A SPECTRAL SEQUENCE FOR L-SPACE LINKS}

In this section we review some material from [GN15]. Given $u, v \in \mathbb{Z}^{r}$, we write $u \preceq v$ if $u_{i} \leq v_{i}$ for all $i$, and $u \prec v$ if $u \preceq v$ and $u \neq v$. Recall that we work with $\mathbb{F}=\mathbb{Z} / 2 \mathbb{Z}$ coefficients.

Definition 3.1. Given a $r$-component oriented link $L$, we define an affine lattice over $\mathbb{Z}^{r}$ :

$$
\mathbb{H}(L)=\bigoplus_{i=1}^{r} \mathbb{H}_{i}(L), \quad \mathbb{H}_{i}(L)=\mathbb{Z}+\frac{1}{2} \operatorname{lk}\left(L_{i}, L-L_{i}\right)
$$

Let us recall that the Heegaard-Floer complex for a $r$-component link $L$ is naturally filtered by the subcomplexes $A_{L}^{-}(L ; v)$ of $\mathbb{F}\left[U_{1}, \ldots, U_{r}\right]$-modules for $v \in \mathbb{H}(L)$. Such a subcomplex is spanned by the generators in the Heegaard-Floer complex of Alexander filtration less than or equal to $v$ in the natural partial order on $\mathbb{H}(L)$. The group $\operatorname{HFL}^{-}(L, v)$ can be defined as the homology of the associated graded complex:

$$
\operatorname{HFL}^{-}(L, v)=H_{*}\left(A^{-}(L ; v) / \sum_{u \prec v} A^{-}(L ; u)\right) .
$$

One can forget a component $L_{r}$ in $L$ and consider the $(r-1)$-component link $L-L_{r}$. There is a natural forgetful map $\pi_{r}: \mathbb{H}(L) \rightarrow \mathbb{H}\left(L-L_{r}\right)$ defined by the equation:

$$
\pi_{r}\left(v_{1}, \ldots, v_{r}\right)=\left(v_{1}-\operatorname{lk}\left(L_{1}, L_{r}\right) / 2, \ldots, v_{r-1}-\operatorname{lk}\left(L_{r-1}, L_{r}\right) / 2\right) .
$$

Similarly, one can define a map $\pi_{L^{\prime}}: \mathbb{H}(L) \rightarrow \mathbb{H}\left(L^{\prime}\right)$ for every sublink $L^{\prime} \subset L$. Furthermore, for large $v_{r} \gg 0$ the subcomplexes $A^{-}(L ; v)$ stabilize, and by [OS08, Proposition 7.1] one has a natural homotopy equivalence $A^{-}(L ; v) \sim A^{-}\left(L-L_{r} ; \pi_{r}(v)\right)$. More generally, for a sublink $L^{\prime}=L_{i_{1}} \cup \ldots \cup L_{i_{r^{\prime}}}$ one gets

$$
A^{-}\left(L^{\prime} ; \pi_{L^{\prime}}(v)\right) \sim A^{-}(L ; v), \text { if } v_{i} \gg 0 \text { for } i \notin\left\{i_{1} \ldots, i_{r^{\prime}}\right\} .
$$

We will use the "inversion theorem" of [GN15], expressing the $h$-function of a link in terms of the Alexander polynomials of its sublinks, or, equivalently, the Euler characteristics of their Heegaard-Floer homology. Define $\chi_{L, v}:=\chi\left(\operatorname{HFL}^{-}(L, v)\right)$. Then by [OS08]

$$
\chi_{L}\left(t_{1}, \ldots, t_{r}\right):=\sum_{v \in \mathbb{H}(L)} \chi_{L, v} t_{1}^{v_{1}} \cdots t_{r}^{v_{r}}= \begin{cases}\left(t_{1} \cdots t_{r}\right)^{1 / 2} \Delta\left(t_{1}, \ldots, t_{r}\right), & \text { if } r>1 \\ \Delta(t) /\left(1-t^{-1}\right), & \text { if } r=1,\end{cases}
$$

where $\Delta\left(t_{1}, \ldots, t_{r}\right)$ denotes the symmetrized Alexander polynomial.

Remark 3.2. We choose the factor $\left(t_{1} \cdots t_{r}\right)^{1 / 2}$ to match more established conventions on the gradings for the hat-version of link Floer homology. For example, the Alexander polynomial of the Hopf link equals 1, and one can check [OS08] that $\widehat{\mathrm{HFL}}$ is supported in Alexander degrees $\left( \pm \frac{1}{2}, \pm \frac{1}{2}\right)$. Since the maximal Alexander degrees in $\widehat{\mathrm{HFL}}$ and $\mathrm{HFL}^{-}$coincide, one gets $\chi_{T(2,2)}\left(t_{1}, t_{2}\right)=t_{1}^{1 / 2} t_{2}^{1 / 2}$.

The following "large surgery theorem" underlines the importance of $A^{-}(L ; v)$. 
Theorem 3.3 ([MO10]). The homology of $A^{-}(L ; v)$ is isomorphic to the Heegaard-Floer homology

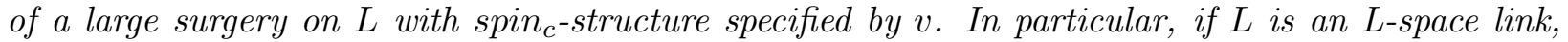
then $H_{*}\left(A^{-}(L, v)\right) \simeq \mathbb{F}[U]$ for all $v$ and all $U_{i}$ are homotopic to each other on the subcomplex $A^{-}(L ; v)$.

One can show that for L-space links the inclusion $h_{v}: A^{-}(L, v) \hookrightarrow A^{-}\left(S^{3}\right)$ is injective on homology, so it is multiplication by $U^{h_{L}(v)}$. Therefore the generator of $H_{*}\left(A^{-}(L, v)\right) \simeq \mathbb{F}[U]$ has homological degree $-2 h_{L}(v)$. The function $h_{L}(v)$ will be called the $h$-function for an L-space link $L$. In [GN15] it was called an "HFL-weight function".

Furthermore, if $L$ is an L-space link, then for large $N \in \mathbb{H}(L)$ one has

$$
\chi\left(A^{-}(L ; N) / A^{-}(L, v)\right)=h_{L}(v) .
$$

Hence, by (3.1) and the inclusion-exclusion formula one can write:

$$
\chi_{L, v}=\sum_{B \subset\{1, \ldots, r\}}(-1)^{|B|-1} h_{L}\left(v-e_{B}\right),
$$

where $e_{B}$ denotes the characteristic vector of the subset $B \subset\{1, \ldots, r\}$. Furthermore, by (3.2) for a sublink $L^{\prime}=L_{i_{1}} \cup \ldots \cup L_{i_{r^{\prime}}}$ one gets

$$
h_{L^{\prime}}\left(\pi_{L^{\prime}}(v)\right)=h_{L}(v) \text {, if } v_{i} \gg 0 \text { for } i \notin\left\{i_{1} \ldots, i_{r^{\prime}}\right\} .
$$

For $r=1$ equation (3.3) has the form $\chi_{L, v}=h(v-1)-h(v)$, so $h(v)$ can be easily reconstructed from the Alexander polynomial: $h_{L}(v)=\sum_{u \geq v+1} \chi_{L, v}$. For $r>1$, one can also show that equation (3.3) (together with the boundary conditions (3.4)) has a unique solution, which is given by the following theorem:

Theorem 3.4 ([GN15]). The h-function of an L-space link is determined by the Alexander polynomials of its sublinks as following:

$$
h_{L}\left(v_{1}, \ldots, v_{r}\right)=\sum_{L^{\prime} \subseteq L}(-1)^{r^{\prime}-1} \sum_{u \succeq \pi_{L^{\prime}}(v+\mathbf{1})} \chi_{L^{\prime}, u},
$$

where the sublink $L^{\prime}$ has $r^{\prime}$ components and $\mathbf{1}=(1, \ldots, 1)$.

Given an L-space link, we construct a spectral sequence whose $E_{2}$ page can be computed from the multi-variable Alexander polynomial by an explicit combinatorial procedure, and whose $E_{\infty}$ page coincides with the group $\mathrm{HFL}^{-}$. The complex (3.1) is quasi-isomorphic to the iterated cone:

$$
\mathcal{K}(v)=\bigoplus_{B \subset\{1, \ldots, r\}} A^{-}\left(L, v-e_{B}\right),
$$

where the differential consists of two parts: the first acts in each summand and the second acts by inclusion maps between summands. There is a spectral sequence naturally associated to this construction. Its $E_{1}$ term equals

$$
E_{1}(v)=\bigoplus_{B \subset\{1 \ldots, r\}} H_{*}\left(A^{-}\left(L, v-e_{B}\right)\right)=\bigoplus_{B \subset\{1 \ldots, r\}} \mathbb{F}[U]\left\langle z\left(v-e_{B}\right)\right\rangle,
$$

where $z(u)$ is the generator of $H_{*}\left(A^{-}(L, u)\right)$ of degree $-2 h_{L}(u)$. The next differential $\partial_{1}$ is induced by inclusions and reads as:

$$
\partial_{1}\left(z\left(v-e_{B}\right)\right)=\sum_{i \in B} U^{h\left(v-e_{B}\right)-h\left(v-e_{B-i}\right)} z\left(v-e_{B}+e_{i}\right)
$$

We obtain the following result. 
Theorem 3.5 ([GN15]). Let $L$ be an L-space link with $r$ components and let $h_{L}(v)$ be the corresponding $h$-function. Then there is a spectral sequence with $E_{2}(v)=H_{*}\left(E_{1}, \partial_{1}\right)$ and $E_{\infty} \simeq$ $\operatorname{HFL}^{-}(L, v)$.

Remark 3.6. Let us write more precisely the bigrading on the $E_{2}$ page. The $E_{1}$ page is naturally bigraded as follows: a generator $U^{m} z\left(v-e_{B}\right)$ has cube degree $|B|$ and its homological degree in $A^{-}\left(L, v-e_{B}\right)$ equals $-2 m-2 h\left(v-e_{B}\right)$. In short, we will write

$$
\operatorname{bideg}\left(U^{m} z\left(v-e_{B}\right)\right)=\left(|B|,-2 m-2 h\left(v-e_{B}\right)\right) \text {. }
$$

The homological degree of the same generator in $E_{1}(v)$ equals the sum of these two degrees. The differential $\partial_{1}$ has bidegree $(-1,0)$, and, more generally, the differential $\partial_{k}$ in the spectral sequence has bidegree $(-k, k-1)$.

In the next section we will compute the $E_{2}$ page for cable L-space links and show that $E_{2}=E_{\infty}$. Let us discuss the action of the operators $U_{i}$ on the $E_{2}$ page. Recall that $U_{i}$ maps $A^{-}(L, v)$ to $A^{-}\left(L, v-e_{i}\right)$, and in homology one has:

$$
U_{i} z(v)=U^{1-h\left(v-e_{i}\right)+h(v)} z\left(v-e_{i}\right) .
$$

Since $U_{i}$ commutes with the inclusions of various $A^{-}$, we get the following result.

Proposition 3.7. Equation (3.7) defines a chain map from $\mathcal{K}(v)$ to $\mathcal{K}\left(v-e_{i}\right)$ commuting with the differential $\partial_{1}$, so we have a well-defined combinatorial map

$$
U_{i}: H_{*}\left(E_{1}(v), \partial_{1}\right) \rightarrow H_{*}\left(E_{1}\left(v-e_{i}\right), \partial_{1}\right) .
$$

If $E_{2}=E_{\infty}$ then one obtains $U_{i}: \operatorname{HFL}^{-}(L, v) \rightarrow \operatorname{HFL}^{-}\left(L, v-e_{i}\right)$.

Furthermore, by the definition of $\widehat{\mathrm{HFL}}[\mathrm{OS} 08$, Section 4] one gets:

$$
\widehat{\operatorname{HFL}}(L, v)=H_{*}\left(A^{-}(L, v) /\left[\sum_{i=1}^{r} A^{-}\left(v-e_{i}\right) \oplus \sum_{i=1}^{r} U_{i} A^{-}\left(v+e_{i}\right)\right]\right) .
$$

This implies the following result:

Proposition 3.8. There is a spectral sequence with $E_{1}$ page

$$
\widehat{E}_{1}=\bigoplus_{B \subset\{1, \ldots, r\}} \operatorname{HFL}^{-}\left(L, v+e_{B}\right)
$$

and converging to $\widehat{E}_{\infty}=\widehat{\operatorname{HFL}}(L, v)$. The differential $\widehat{\partial}_{1}$ is given by the action of $U_{i}$ induced by (3.7).

\section{HeEgaARD-Floer homology FOR CABle LiNKS}

4.1. The Alexander polynomial and $h$-function. The Alexander polynomial of cable knots and links is given by the following well-known formula:

$$
\Delta_{K_{r m, r n}}\left(t_{1}, \ldots, t_{r}\right)=\Delta_{K}\left(t_{1}^{m} \cdots t_{r}^{m}\right) \cdot \Delta_{T(r m, r n)}\left(t_{1}, \ldots, t_{r}\right),
$$

where $T(r m, r n)$ denotes the $(r m, r n)$ torus link. Throughout, let $\mathbf{t}=t_{1} \cdots t_{r}$ and $l=m n$.

Lemma 4.1. The generating functions for the Euler characteristics of $\mathrm{HFL}^{-}$for $K_{r m, r n}$ and $K_{m, n}$ are related by the following equation:

$$
\chi_{K_{r m, r n}}\left(t_{1}, \ldots, t_{r}\right)=\chi_{K_{m, n}}(\mathbf{t}) \cdot\left(\mathbf{t}^{l / 2}-\mathbf{t}^{-l / 2}\right)^{r-1} .
$$


Proof. The statement follows from the identity (4.1) and the expression for the Alexander polynomials of torus links:

$$
\chi_{T(r m, r n)}\left(t_{1}, \ldots, t_{r}\right)=\frac{\left(\mathbf{t}^{m n / 2}-\mathbf{t}^{-m n / 2}\right)^{r}}{\left(\mathbf{t}^{m / 2}-\mathbf{t}^{-m / 2}\right)\left(\mathbf{t}^{n / 2}-\mathbf{t}^{-n / 2}\right)} .
$$

Remark 4.2. The Alexander polynomial is determined up to a sign. By (4.2), the multivariable Alexander polynomial of a cable link is supported on the diagonal, so one can fix the sign by requiring its top coefficient to be positive.

From now on we will assume that $K$ is an L-space knot and $n / m \geq 2 g(K)-1$, so $K_{r m, r n}$ is an L-space link for all $r$. To simplify notation, we define $h_{r m, r n}(v)=h_{K_{r m, r n}}(v)$ and $\chi_{r m, r n}(v)=$ $\chi_{K_{r m, r n}, v}$. Let $c=l(r-1) / 2$.

Theorem 4.3. Suppose that $v_{1} \leq v_{2} \leq \ldots \leq v_{r}$. Then the following equation holds:

$$
h_{r m, r n}\left(v_{1}, \ldots, v_{r}\right)=h_{m, n}\left(v_{1}-c\right)+h_{m, n}\left(v_{2}-c+l\right)+\ldots+h_{m, n}\left(v_{r}-c+(r-1) l\right) .
$$

Proof. We will use Theorem 3.4 to compute $h(v)$. Let $L^{\prime}$ be a sublink of $K_{r m, r n}$ with $r^{\prime}$ components, i.e., $L^{\prime}=K_{r^{\prime} m, r^{\prime} n}$. By (4.2), one has

$$
\chi_{K_{r^{\prime} m, r^{\prime} n}}\left(t_{1}, \ldots, t_{r^{\prime}}\right)=\chi_{K_{m, n}}(\mathbf{t}) \cdot \mathbf{t}^{l\left(r^{\prime}-1\right) / 2} \sum_{j=0}^{r^{\prime}-1}(-1)^{j}\left(\begin{array}{c}
r^{\prime}-1 \\
j
\end{array}\right) \mathbf{t}^{-l j},
$$

hence $\chi_{L^{\prime}, u}$ does not vanish only if $u=(s, \ldots, s)$, and

$$
\chi_{L^{\prime}, s, \ldots, s}=\sum_{j=0}^{r^{\prime}-1}(-1)^{j}\left(\begin{array}{c}
r^{\prime}-1 \\
j
\end{array}\right) \chi_{m, n}\left(s-l\left(r^{\prime}-1\right) / 2+l j\right) .
$$

Therefore

$$
\begin{aligned}
\sum_{u \succeq \pi_{L^{\prime}}(v+\mathbf{1})} \chi_{L^{\prime}, u} & =\sum_{s>\max \left(\pi_{L^{\prime}}(v)\right)} \sum_{j=0}^{r^{\prime}-1}(-1)^{j}\left(\begin{array}{c}
r^{\prime}-1 \\
j
\end{array}\right) \chi_{m, n}\left(s-l\left(r^{\prime}-1\right) / 2+l j\right) \\
& =\sum_{j=0}^{r^{\prime}-1}(-1)^{j}\left(\begin{array}{c}
r^{\prime}-1 \\
j
\end{array}\right) h_{m, n}\left(\max \left(\pi_{L^{\prime}}(v)\right)-l\left(r^{\prime}-1\right) / 2+l j\right) .
\end{aligned}
$$

Furthermore, if $L^{\prime}=L_{i_{1}} \cup \ldots \cup L_{i_{r^{\prime}}}$ then $\pi_{L^{\prime}}(v)=\left(v_{i_{1}}-l\left(r-r^{\prime}\right) / 2, \ldots, v_{i_{r^{\prime}}}-l\left(r-r^{\prime}\right) / 2\right)$, so

$$
\max \left(\pi_{L^{\prime}}(v)\right)=\max \left(v_{i_{1}}, \ldots, v_{i_{r}^{\prime}}\right)-l\left(r-r^{\prime}\right) / 2=\max \left(v_{L^{\prime}}\right)-l\left(r-r^{\prime}\right) / 2 .
$$

This means that (3.5) can be rewritten as follows:

$$
\begin{aligned}
h_{r m, r n}\left(v_{1}, \ldots, v_{r}\right) & =\sum_{L^{\prime}, j}(-1)^{r^{\prime}-1+j}\left(\begin{array}{c}
r^{\prime}-1 \\
j
\end{array}\right) h_{m, n}\left(\max \left(v_{L^{\prime}}\right)-l(r-1) / 2+l j\right) \\
& =\sum_{i, j} h_{m, n}\left(v_{i}-l(r-1) / 2+l j\right) \sum_{L^{\prime}: v_{i}=\max \left(v_{L^{\prime}}\right)}(-1)^{r^{\prime}-1+j}\left(\begin{array}{c}
r^{\prime}-1 \\
j
\end{array}\right) .
\end{aligned}
$$

One can check that the inner sum vanishes unless $j=i-1$ (recall that $v_{1} \leq v_{2} \leq \ldots \leq v_{r}$ ), so one gets

$$
h_{r m, r n}\left(v_{1}, \ldots, v_{r}\right)=\sum_{i} h_{m, n}\left(v_{i}-l(r-1) / 2+l(i-1)\right) .
$$


Lemma 4.4. The following identity holds:

$$
h_{r m, r n}\left(-v_{1}, \ldots,-v_{r}\right)=h_{r m, r n}\left(v_{1}, \ldots, v_{r}\right)+\left(v_{1}+\ldots+v_{r}\right) .
$$

Proof. Suppose that $v_{1} \leq v_{2} \leq \ldots \leq v_{r}$. Then $-v_{1} \geq-v_{2} \geq \ldots \geq-v_{r}$. Therefore

$$
\begin{aligned}
h_{r m, r n}\left(-v_{1}, \ldots,-v_{r}\right) & =\sum_{i=1}^{r} h_{m, n}\left(-v_{i}-l(r-1) / 2+l(r-i)\right) \\
& =\sum_{i=1}^{r} h_{m, n}\left(-v_{i}+l(r-1) / 2-l(i-1)\right) .
\end{aligned}
$$

It is known (e.g., [HLZ13]) that for all $x$,

$$
h_{m, n}(-x)=h_{m, n}(x)+x,
$$

hence

$$
h_{m, n}\left(-v_{i}+l(r-1) / 2-l(i-1)\right)=h_{m, n}\left(v_{i}-l(r-1) / 2+l(i-1)\right)+\left(v_{i}-l(r-1) / 2+l(i-1)\right) .
$$

Finally, $\sum_{i=1}^{r}(-l(r-1) / 2+l(i-1))=0$.

Lemma 4.5. One has $h_{r m, r n}(k, k \ldots, k)=\mathbf{h}(k)$, where $\mathbf{h}(k)$ is defined by (1.1).

Proof. Indeed, by (4.3) we have

$$
h_{r m, r n}(k, \ldots, k)=h_{m, n}(k-l(r-1) / 2)+h_{m, n}(k-l(r-1) / 2+l)+\ldots+h_{m, n}(k+l(r-1) / 2),
$$

SO

$$
\sum_{k} h_{r m, r n}(k, \ldots, k) t^{k}=\left(t^{-l(r-1) / 2}+\ldots+t^{l(r-1) / 2}\right) \sum_{k} h_{m, n}(k) t^{k}=\frac{\left(t^{l r / 2}-t^{-l r / 2}\right)}{\left(t^{l / 2}-t^{-l / 2}\right)} \cdot \frac{t^{-1} \Delta_{m, n}(t)}{\left(1-t^{-1}\right)^{2}} .
$$

For the rest of this section we will assume that $n / m>2 g(K)-1$.

Lemma 4.6. If $v \leq g\left(K_{m, n}\right)-l$, then $\operatorname{HFK}^{-}\left(K_{m, n}, v\right) \simeq \mathbb{F}$.

Proof. By [Hed09, Theorem 1.10], $K_{m, n}$ is an L-space knot and hence by [OS05]

$$
g\left(K_{m, n}\right)=\tau\left(K_{m, n}\right), \quad g(K)=\tau(K) .
$$

By [Shi85], we have:

$$
g\left(K_{m, n}\right)=m g(K)+\frac{(m-1)(n-1)}{2},
$$

so for $n / m>2 g(K)-1$ we have

$$
2 g\left(K_{m, n}\right)=2 m g(K)+m n-m-n+1<m n+1,
$$

hence $l=m n \geq 2 g\left(K_{m, n}\right)$. On the other hand, it is well-known that for $v \leq-g\left(K_{m, n}\right)$ one has $\left.\operatorname{HFK}^{-}\left(K_{m, n}, v\right)\right) \simeq \mathbb{F}$.

We will use the function $\beta$ defined by (1.1).

Lemma 4.7. If $\beta(k)=-1$ then $\operatorname{HFK}^{-}\left(K_{m, n}, k-c\right)=0$. Otherwise

$$
\beta(k)=\max \left\{j: 0 \leq j \leq r-1, \operatorname{HFK}^{-}\left(K_{m, n}, k-c+l j\right) \simeq \mathbb{F}\right\} .
$$


Proof. By (1.1) and Lemma 4.5 we have

$\beta(k)+1=h_{r m, r n}(k-1, \ldots, k-1)-h_{r m, r n}(k, \ldots, k)=\sum_{j=0}^{r-1}\left(h_{m, n}(k-1-c+l j)-h_{m, n}(k-c+l j)\right)$.

Note that $h_{m, n}(k-1-c+l j)-h_{m, n}(k-c+l j)=\operatorname{dim} \operatorname{HFK}^{-}\left(K_{m, n}, k-c+l j\right) \in\{0,1\}$. If $\operatorname{HFK}^{-}\left(K_{m, n}, k-c+l j\right) \simeq \mathbb{F}$ then $k-c+l j \leq g\left(K_{m, n}\right)$, so by Lemma $4.6 \operatorname{HFK}^{-}\left(K_{m, n}, k-c+l j^{\prime}\right) \simeq \mathbb{F}$ for all $j^{\prime}<j$. Therefore, if $\operatorname{HFK}^{-}\left(K_{m, n}, k-c\right)=0$ then $\beta(k)=-1$, otherwise

$$
\operatorname{HFK}^{-}\left(K_{m, n}, k-c+l j\right)= \begin{cases}\mathbb{F} & \text { if } j \leq \beta(k) \\ 0 & \text { if } j>\beta(k)\end{cases}
$$

Suppose that $v_{1}=\ldots=v_{\lambda_{1}}=u_{1}, v_{\lambda_{1}+1}=\ldots=v_{\lambda_{1}+\lambda_{2}}=u_{2}, \ldots, v_{\lambda_{1}+\ldots+\lambda_{s-1}+1}=\ldots=v_{r}=u_{s}$ where $u_{1}<u_{2}<\ldots<u_{s}$ and $\lambda_{1}+\ldots+\lambda_{s}=r$. We will abbreviate this as $v=\left(u_{1}^{\lambda_{1}}, \ldots, u_{s}^{\lambda_{s}}\right)$.

Lemma 4.8. Suppose that $\beta\left(u_{s}\right)<r-\lambda_{s}$. Then for any subset $B \subset\{1, \ldots, r-1\}$ one has $h_{r m, r n}\left(v-e_{B}\right)=h_{r m, r n}\left(v-e_{B}-e_{r}\right)$.

Proof. To apply (4.3), one needs to reorder the components of the vectors $v-e_{B}$ and $v-e_{B}-e_{r}$. Note that in both cases the last (largest) $\lambda_{s}$ components are equal either to $u_{s}$ or to $u_{s}-1$, and the corresponding contributions to $h_{r m, r n}$ are equal to $h_{m, n}\left(u_{s}-c+l\left(r-\lambda_{s}\right)+l j\right)$ or to $h_{m, n}\left(u_{s}-c+l\left(r-\lambda_{s}\right)+l j-1\right)$, respectively $\left(j=0, \ldots, \lambda_{s}-1\right)$. On the other hand, by (4.4) one has

$$
\operatorname{HFK}^{-}\left(K_{m, n}, u_{s}-c+l\left(r-\lambda_{s}\right)+l j\right)=0
$$

and so

$$
h_{m, n}\left(u_{s}-c+l\left(r-\lambda_{s}\right)+l j-1\right)=h_{m, n}\left(u_{s}-c+l\left(r-\lambda_{s}\right)+l j\right) .
$$

Lemma 4.9. If $\beta\left(u_{s}\right) \geq r-\lambda_{s}$ then $h_{r m, r n}(v)=\mathbf{h}\left(u_{s}\right)+r u_{s}-|v|$.

Proof. Since $\beta\left(u_{s}\right) \geq r-\lambda_{s}$, we have $\operatorname{HFK}^{-}\left(K_{m, n}, u_{s}-c+l\left(r-\lambda_{s}\right)\right) \simeq \mathbb{F}$, so

$$
u_{s}-c+l\left(r-\lambda_{s}\right) \leq g\left(K_{m, n}\right) .
$$

For $i \leq r-\lambda_{s}$ we get

$$
v_{i}-c+l(i-1)<u_{s}-c+l(i-1) \leq u_{s}-c+l\left(r-\lambda_{s}\right)-l \leq g\left(K_{m, n}\right)-l,
$$

so by Lemma 4.6, $\operatorname{HFK}^{-}\left(K_{m, n}, w\right) \simeq \mathbb{F}$ for all $w \in\left[v_{i}-c+l(i-1), u_{s}-c+l(i-1)\right]$, and

$$
h_{m, n}\left(v_{i}-c+l(i-1)\right)=h_{m, n}\left(u_{s}-c+l(i-1)\right)+\left(u_{s}-v_{i}\right) .
$$

Now the statement follows from Lemma 4.3.

Lemma 4.10. Suppose that $\beta\left(u_{s}\right) \geq r-\lambda_{s}$. Then for any subsets $B^{\prime} \subset\left\{1, \ldots, r-\lambda_{s}\right\}$ and $B^{\prime \prime} \subset\left\{r-\lambda_{s}+1, \ldots, r\right\}$ one has

$$
h_{r m, r n}\left(v-e_{B^{\prime}}-e_{B^{\prime \prime}}\right)=h_{r m, r n}(v)+\left|B^{\prime}\right|+\min \left(\left|B^{\prime \prime}\right|, \beta\left(u_{s}\right)-r+\lambda_{s}+1\right) .
$$


Proof. Since $\mathrm{HFK}^{-}\left(K_{m, n}, u_{s}-c+l\left(r-\lambda_{s}\right)\right) \simeq \mathbb{F}$, we have $u_{s}-c+l\left(r-\lambda_{s}\right) \leq g\left(K_{m, n}\right)$, so for all $i \leq r-\lambda_{s}$ one has $v_{i}-c+l(i-1)<u_{s}-c+l\left(r-\lambda_{s}\right)-l \leq g\left(K_{m, n}\right)-l$, and by Lemma 4.6 $\operatorname{HFK}^{-}\left(K_{m, n}, v_{i}-c+l(i-1)\right) \simeq \mathbb{F}$, and $h_{m, n}\left(v_{i}-1-c+l(i-1)\right)=h_{m, n}\left(v_{i}-c+l(i-1)\right)+1$. Therefore $h_{r m, r n}\left(v-e_{B^{\prime}}-e_{B^{\prime \prime}}\right)=\left|B^{\prime}\right|+h_{r m, r n}\left(v-e_{B^{\prime \prime}}\right)$. Finally,

$$
\begin{aligned}
h_{r m, r n}\left(v-e_{B^{\prime \prime}}\right)-h_{r m, r n}(v) & =\sum_{j=0}^{\left|B^{\prime \prime}\right|}\left(h_{m, n}\left(u_{s}-1-c+l\left(r-\lambda_{s}\right)+l j\right)-h_{m, n}\left(u_{s}-c+l\left(r-\lambda_{s}\right)+l j\right)\right. \\
& =\min \left(\left|B^{\prime \prime}\right|, \beta\left(u_{s}\right)-r+\lambda_{s}+1\right) .
\end{aligned}
$$

\subsection{Spectral sequence for $\mathrm{HFL}^{-}$.}

Definition 4.11. Let $\mathcal{E}_{r}$ denote the exterior algebra over $\mathbb{F}$ with variables $z_{1}, \ldots, z_{r}$. Let us define the cube differential on $\mathcal{E}_{r}$ by the equation

$$
\partial\left(z_{\alpha_{1}} \wedge \ldots \wedge z_{\alpha_{k}}\right)=\sum_{j=1}^{k} z_{\alpha_{1}} \wedge \ldots \wedge \widehat{z_{\alpha_{j}}} \wedge \ldots \wedge z_{\alpha_{k}},
$$

and the $b$-truncated differential on $\mathcal{E}_{r}[U]$ by the equation

$$
\partial^{(b)}\left(z_{\alpha_{1}} \wedge \ldots \wedge z_{\alpha_{k}}\right)= \begin{cases}U \partial\left(z_{\alpha_{1}} \wedge \ldots \wedge z_{\alpha_{k}}\right), & \text { if } k \leq b \\ \partial\left(z_{\alpha_{1}} \wedge \ldots \wedge z_{\alpha_{k}}\right), & \text { if } k>b .\end{cases}
$$

More invariantly, one can define the weight of a monomial $z_{\alpha}=z_{\alpha_{1}} \wedge \ldots \wedge z_{\alpha_{k}}$ as $w\left(z_{\alpha}\right)=$ $\min (|\alpha|, b)$, and the $b$-truncated differential is given by the equation:

$$
\partial^{(b)}\left(z_{\alpha}\right)=\sum_{i \in \alpha} U^{w(\alpha)-w\left(\alpha-\alpha_{i}\right)} z_{\alpha-\alpha_{i}} .
$$

Indeed, $w(\alpha)-w\left(\alpha-\alpha_{i}\right)=1$ for $|\alpha| \leq b$ and $w(\alpha)-w\left(\alpha-\alpha_{i}\right)=0$ for $|\alpha|>b$.

Definition 4.12. Let $\mathcal{E}_{r}^{\text {red }} \subset \mathcal{E}_{r}$ be the subalgebra of $\mathcal{E}_{r}$ generated by the differences $z_{i}-z_{j}$ for all $i \neq j$.

Lemma 4.13. The kernel of the cube differential $\partial$ on $\mathcal{E}_{r}$ coincides with $\mathcal{E}_{r}^{\text {red }}$.

Proof. It is clear that $\partial\left(z_{i}-z_{j}\right)=0$, and Leibniz rule implies vanishing of $\partial$ on $\mathcal{E}_{r}^{\text {red }}$. Let us prove that Ker $\partial \subset \mathcal{E}_{r}^{\text {red }}$. Since $\left(\mathcal{E}_{r}, \partial\right)$ is acyclic, it is sufficient to prove that the image of every monomial $z_{\alpha_{1}} \wedge \cdots \wedge z_{\alpha_{k}}$ is contained in $\mathcal{E}_{r}$. Indeed, one can check that

$$
\partial\left(z_{\alpha_{1}} \wedge \cdots \wedge z_{\alpha_{k}}\right)=\left(z_{\alpha_{2}}-z_{\alpha_{1}}\right) \wedge \cdots \wedge\left(z_{\alpha_{k}}-z_{\alpha_{k-1}}\right) \text {. }
$$

Lemma 4.14. The homology of $\partial^{(b)}$ is given by the following equation:

$$
\operatorname{dim} H_{k}\left(\mathcal{E}_{r}[U], \partial^{(b)}\right)= \begin{cases}\left(\begin{array}{c}
r-1 \\
k
\end{array}\right), & \text { if } k<b \\
0, & \text { if } k \geq b .\end{cases}
$$

Proof. Since $\partial$ is acyclic, one immediately gets $H_{k}\left(\mathcal{E}_{r}[U], \partial^{(b)}\right)=0$ for $k \geq b$. For $k<b$, the homology is supported at the zeroth power of $U$ and one has $H_{k}\left(\mathcal{E}_{r}[U]\right) \simeq \operatorname{Ker}\left(\left.\partial\right|_{\wedge^{k}\left(z_{1}, \ldots, z_{r}\right)}\right)$. The dimension of the latter kernel equals

$$
\operatorname{dim} \operatorname{Ker}\left(\left.\partial\right|_{\wedge^{k}\left(z_{1}, \ldots, z_{r}\right)}\right)=\operatorname{dim} \wedge^{k}\left(z_{1}-z_{2}, \ldots, z_{1}-z_{r}\right)=\left(\begin{array}{c}
r-1 \\
k
\end{array}\right) .
$$


Proof of Theorem 5. Let us compute $\operatorname{HFL}^{-}\left(K_{r m, r n}, v\right)$ using the spectral sequence constructed in Theorem 3.5. By Lemma 4.8, in case (a) it is easy to see that the complex $\left(E_{1}, \partial_{1}\right)$ is contractible in the direction of $e_{r}$ and $E_{2}=H_{*}\left(E_{1}, \partial_{1}\right)=0$.

In case (b) by Lemma 4.10 and (4.5) one can write $E_{1}=\mathcal{E}_{r-\lambda_{s}}[U] \otimes_{\mathbb{F}[U]} \mathcal{E}_{\lambda_{s}}[U]$, a tensor product of chain complexes of $\mathbb{F}[U]$-modules, and $\partial_{1}$ acts as $U \partial$ on the first factor and as $\partial^{(\beta+1)}$ on the second one. This implies

$$
E_{2}=H_{*}\left(E_{1}, \partial_{1}\right) \simeq \mathcal{E}_{r-\lambda_{s}} \otimes_{\mathbb{F}} H_{*}\left(\mathcal{E}_{\lambda_{s}}[U], \partial^{(\beta+1)}\right) .
$$

Indeed, $U$ acts trivially on $H_{*}\left(\mathcal{E}_{\lambda_{s}}[U], \partial^{(\beta+1)}\right)$, so one can take the homology of $\partial^{(\beta+1)}$ first and then observe that $U \partial$ vanishes on

$$
\mathcal{E}_{r-\lambda_{s}}[U] \otimes_{\mathbb{F}[U]} H_{*}\left(\mathcal{E}_{\lambda_{s}}[U], \partial^{(\beta+1)}\right) \simeq \mathcal{E}_{r-\lambda_{s}} \otimes_{\mathbb{F}} H_{*}\left(\mathcal{E}_{\lambda_{s}}[U], \partial^{(\beta+1)}\right) .
$$

By Lemma 4.14, the $E_{2}$ page (4.6) agrees with the statement of the theorem, hence we need to prove that the spectral sequence collapses.

Indeed, the $E_{1}$ page is bigraded by the homological degree and $|B|$ (see Remark 3.6). By Lemma 4.14 any surviving homology class on the $E_{2}$ page of cube degree $x$ has bidegree $\left(x,-2 h_{r m, r n}(v)-\right.$ $2 x)$, so all bidegrees on the $E_{2}$ page belong to the same line of slope $(-2)$. Therefore all higher differentials must vanish.

Finally, a simple formula for $h_{r m, r n}(v)$ in case (b) follows from Lemma 4.9.

4.3. Action of $U_{i}$. One can use Proposition 3.7 to compute the action of $U_{i}$ on $\mathrm{HFL}^{-}$for cable links. Recall that $R=\mathbb{F}\left[U_{1} \ldots, U_{r}\right]$. Throughout this section we assume $n / m>2 g(K)-1$. We start with a simple algebraic statement.

Proposition 4.15. Let $\mathcal{C}$ be an $\mathbb{F}$-algebra. Given a finite collection of elements $c_{\alpha} \in \mathcal{C}$ and vectors $v^{(\alpha)} \in \mathbb{Z}^{r}$, consider the ideal $\mathcal{I} \subset \mathcal{C} \otimes_{\mathbb{F}} R$ generated by $c_{\alpha} \otimes U_{1}^{v_{1}^{(\alpha)}} \cdots U_{r}^{v_{r}^{(\alpha)}}$. Then the following statements hold:

(a) The quotient $\left(\mathcal{C} \otimes_{\mathbb{F}} R\right) / \mathcal{I}$ can be equipped with a $\mathbb{Z}^{r}$-grading, with $U_{i}$ of grading $\left(-e_{i}\right)$ and $\mathcal{C}$ of grading 0.

(b) The subspace of $\left(\mathcal{C} \otimes_{\mathbb{F}} R\right) / \mathcal{I}$ with grading $v$ is isomorphic to

$$
\left[\left(\mathcal{C} \otimes_{\mathbb{F}} R\right) / \mathcal{I}\right](v) \simeq \mathcal{C} /\left(c_{\alpha}: v^{(\alpha)} \preceq-v\right) .
$$

Proof. Straightforward.

Definition 4.16. We define $\mathcal{A}_{r}=\mathcal{E}_{r} \otimes_{\mathbb{F}} R$ and $\mathcal{A}_{r}^{\text {red }}=\mathcal{E}_{r}^{\text {red }} \otimes_{\mathbb{F}} R$. Let $\mathcal{I}_{\beta}^{\prime}$ denote the ideal in $\mathcal{A}_{r}$ generated by the monomials $\left(z_{i_{1}} \wedge \cdots \wedge z_{i_{s}}\right) \otimes U_{i_{s+1}} \cdots U_{i_{\beta+1}}$ for all $s \leq \beta+1$ and all tuples of pairwise distinct $i_{1}, \ldots, i_{\beta+1}$. Let $\mathcal{I}_{\beta}:=\mathcal{I}_{\beta}^{\prime} \cap \mathcal{A}_{r}^{\text {red }}$ be the corresponding ideal in $\mathcal{A}_{r}^{\text {red }}$.

The algebras $\mathcal{A}_{r}$ and $\mathcal{A}_{r}^{\text {red }}$ are naturally $\mathbb{Z}^{r+1}$-graded: the generators $z_{i}$ have Alexander grading 0 and homological grading $(-1)$, the generators $U_{i}$ have Alexander grading $\left(-e_{i}\right)$ and homological grading $(-2)$.

Definition 4.17. We define $\mathcal{H}(k):=\bigoplus_{\max (v) \leq k} \operatorname{HFL}^{-}\left(K_{r m, r n}, v\right)$. Since $U_{i}$ decreases the Alexander grading, $\mathcal{H}(k)$ is naturally an $R$-module.

The following theorem clarifies the algebraic structure of Theorem 5 . 
Theorem 4.18. The following graded $R$-modules are isomorphic:

$$
\mathcal{H}(k) / \mathcal{H}(k-1) \simeq \mathcal{A}_{r}^{\mathrm{red}} / \mathcal{I}_{\beta(k)}[-2 \mathbf{h}(k)]\{k, \ldots, k\},
$$

where [.] and $\{\cdot\}$ denote the shifts of the homological grading and the Alexander grading, respectively.

Proof. By definition, $\mathcal{H}(k) / \mathcal{H}(k-1)$ is supported on the set of Alexander gradings $v$ such that $\max (v)=k$. The monomial $U_{1} \cdots U_{r}$ belongs to the ideal $\mathcal{I}_{\beta(k)}$, so $\mathcal{A}_{r}^{\mathrm{red}} / \mathcal{I}_{\beta(k)}$ is supported on the set of Alexander gradings $u$ with $\max (u)=0$.

Suppose that exactly $\lambda$ components of $v$ are equal to $k$. Without loss of generality we can assume $v_{1}, \ldots, v_{r-\lambda}<k$ and $v_{r-\lambda+1}=\ldots=v_{r}=k$. It follows from Lemma 4.13 and the proof of Theorem 5 that $\operatorname{HFL}^{-}\left(K_{r m, r n}, v\right)$ is isomorphic to the quotient of $\mathcal{E}_{r}^{\text {red }}$ by the ideal generated by degree $\beta-r+\lambda+1$ monomials in $\left(z_{i}-z_{j}\right)$ for $i, j>r-\lambda$.

Consider the subspace of $\mathcal{A}_{r} / \mathcal{I}_{\beta}^{\prime}$ of Alexander grading $\left(v_{1}-k, \ldots, v_{r}-k\right)$. By Proposition 4.15 it is isomorphic to a quotient of $\mathcal{E}_{r}$ modulo the following relations. For each subset $B \subset\{1, \ldots, r-\lambda\}$ and each degree $\beta+1-|B|$ monomial $m^{\prime}$ in variables $z_{i}$ for $i \notin B$ there is a relation $m^{\prime} \otimes \prod_{b \in B} U_{b} \in \mathcal{I}_{\beta}^{\prime}$. All these relations can be multiplied by an appropriate monomial in $R$ to have Alexander grading $\left(v_{1}-k, \ldots, v_{r}-k\right)$.

Note that such $m^{\prime}$ should contain at most $r-\lambda-|B|$ factors with indices in $\{1, \ldots, r-\lambda\} \backslash B$, hence it contains at least $\beta-r+\lambda+1$ factors with indices in $\{r-\lambda+1, \ldots, r\}$. Therefore $\left[\mathcal{A}_{r} / \mathcal{I}_{\beta}^{\prime}\right]\left(v_{1}-k, \ldots, v_{r}-k\right)$ is naturally isomorphic to the quotient of $\mathcal{E}_{r}$ by the ideal generated by degree $\beta-r+\lambda+1$ monomials in $z_{i}$ for $i>r-\lambda$.

We conclude that $\left[\mathcal{A}_{r}^{\text {red }} / \mathcal{I}_{\beta(k)}\right]\left(v_{1}-k, \ldots, v_{r}-k\right)$ is isomorphic to $\operatorname{HFL}^{-}\left(K_{r m, r n}, v\right)$. The action of $U_{i}$ on $\mathcal{H}(k)$ is described by Proposition 3.7. One can check that it commutes with the above isomorphisms for different $v$, so we get the isomorphism of $R$-modules.

We illustrate the above theorem with the following example (cf. Example 5.8).

Example 4.19. Let us describe the subspaces of $\mathcal{A}_{3}^{\text {red }} / \mathcal{I}_{1}$ with various Alexander gradings. The ideal $\mathcal{I}_{1}$ equals:

$$
\mathcal{I}_{1}=\left(\left(z_{1}-z_{2}\right)\left(z_{2}-z_{3}\right),\left(z_{1}-z_{2}\right) U_{3},\left(z_{1}-z_{3}\right) U_{2},\left(z_{2}-z_{3}\right) U_{1}, U_{1} U_{2}, U_{1} U_{3}, U_{2} U_{3}\right) \subset \mathcal{A}_{3}^{\text {red }} .
$$

In the Alexander grading $(0,0,0)$ one gets

$$
\left[\mathcal{A}_{3}^{\text {red }} / \mathcal{I}_{1}\right](0,0,0) \simeq \mathcal{E}_{3}^{\text {red }} /\left(\left(z_{1}-z_{2}\right)\left(z_{2}-z_{3}\right)\right)=\left\langle 1, z_{1}-z_{2}, z_{2}-z_{3}\right\rangle,
$$

in the Alexander grading $(k, 0,0)$ (for $k>0)$ one gets two relations

$$
U_{1}^{k}\left(z_{1}-z_{2}\right)\left(z_{2}-z_{3}\right), U_{1}^{k-1}\left(z_{2}-z_{3}\right) \in \mathcal{I}_{1} .
$$

Since the latter implies the former, we get

$$
\left[\mathcal{A}_{3}^{\text {red }} / \mathcal{I}_{1}\right](k, 0,0) \simeq \mathcal{E}_{3}^{\text {red }} /\left(z_{2}-z_{3}\right)=\left\langle 1, z_{1}-z_{2}\right\rangle .
$$

The map $U_{1}:\left[\mathcal{A}_{3}^{\text {red }} / \mathcal{I}_{1}\right](0,0,0) \rightarrow\left[\mathcal{A}_{3}^{\text {red }} / \mathcal{I}_{1}\right](1,0,0)$ is a natural projection

$$
\mathcal{E}_{3}^{\mathrm{red}} /\left(\left(z_{1}-z_{2}\right)\left(z_{2}-z_{3}\right)\right) \rightarrow \mathcal{E}_{3}^{\mathrm{red}} /\left(z_{2}-z_{3}\right),
$$

while the map $U_{1}:\left[\mathcal{A}_{3}^{\text {red }} / \mathcal{I}_{1}\right](k, 0,0) \rightarrow\left[\mathcal{A}_{3}^{\text {red }} / \mathcal{I}_{1}\right](k+1,0,0)$ is an isomorphism for $k>0$.

The gradings $(0, k, 0)$ and $(0,0, k)$ can be treated similarly. Furthermore, $U_{i} U_{j} \in \mathcal{I}_{1}$ for $i \neq j$, so all other graded subspaces of $\mathcal{A}_{3}^{\text {red }} / \mathcal{I}_{1}$ vanish.

Since the multiplication by $U_{i}$ preserves the ideal $\mathcal{I}_{\beta}$, we get the following useful result. 
Corollary 4.20. If $\max (v)=\max \left(v-e_{i}\right)$, then the map

$$
U_{i}: \operatorname{HFL}^{-}\left(K_{r m, r n}, v\right) \rightarrow \operatorname{HFL}^{-}\left(K_{r m, r n}, v-e_{i}\right)
$$

is surjective.

Lemma 4.21. Suppose that $\max (v)=k$ and $\max \left(v-e_{i}\right)=k-1$, and the homology group $\operatorname{HFL}^{-}\left(K_{r m, r n}, v\right)$ does not vanish. Then $\beta(k)=r-1, \beta(k-1) \geq r-2$ and the map

$$
U_{i}: \operatorname{HFL}^{-}\left(K_{r m, r n}, v\right) \rightarrow \operatorname{HFL}^{-}\left(K_{r m, r n}, v-e_{i}\right)
$$

is surjective.

Proof. Since $\max (v)=k$ and $\max \left(v-e_{i}\right)=k-1$, the multiplicity of $k$ in $v$ equals 1 , so by Theorem $5 \beta(k) \geq r-1$, hence $\beta(k)=r-1$. Therefore $\operatorname{HFL}^{-}\left(K_{r m, r n}, v\right) \simeq \mathcal{E}_{r}^{\text {red }}$, so $U_{i}$ is surjective. Indeed, by Theorem $5 \mathrm{HFL}^{-}\left(K_{r m, r n}, v-e_{i}\right)$ is naturally isomorphic to a quotient of $\mathcal{E}_{r}^{\text {red }}$, and by Proposition $3.7 U_{i}$ coincides with a natural quotient map. Finally, by $(4.4) \operatorname{HFK}^{-}\left(K_{m, n}, k-c+l(r-1)\right) \simeq \mathbb{F}$, and by Lemma $4.6 \operatorname{HFK}^{-}\left(K_{m, n}, k-1-c+l(r-2)\right) \simeq \mathbb{F}$, so $\beta(k-1) \geq r-2$.

Proof of Theorem 6. Let us prove that the homology classes with diagonal Alexander gradings generate $\mathrm{HFL}^{-}$over $R$. Indeed, given $v=\left(v_{1} \leq \ldots \leq v_{r}\right)$ with $\mathrm{HFL}^{-}\left(K_{r m, r n}, v\right) \neq 0$, by Theorems 5 and 4.18 one can check that $\operatorname{HFL}^{-}\left(K_{r m, r n}, v_{r}, \ldots, v_{r}\right) \neq 0$ and by Corollary 4.20 the map

$$
U_{1}^{v_{r}-v_{1}} \cdots U_{r-1}^{v_{r}-v_{r-1}}: \operatorname{HFL}^{-}\left(K_{r m, r n}, v_{r}, \ldots, v_{r}\right) \rightarrow \operatorname{HFL}^{-}\left(K_{r m, r n}, v\right)
$$

is surjective.

Let us describe the $R$-modules generated by the diagonal classes in degree $(k, \ldots, k)$. If $\beta(k)=-1$ then $\operatorname{HFL}^{-}\left(K_{r m, r n}, k, \ldots, k\right)=0$. If $0 \leq \beta(k) \leq r-2$ then by Lemma 4.21 the submodule $R \cdot \operatorname{HFL}^{-}\left(K_{r m, r n}, k, \ldots, k\right)$ does not contain any classes with maximal Alexander degree less than $k$, so by Theorem 4.18

$$
R \cdot \operatorname{HFL}^{-}\left(K_{r m, r n}, k, \ldots, k\right) \simeq \mathcal{A}_{r}^{\mathrm{red}} / \mathcal{I}_{\beta(k)}=: M_{\beta(k)}
$$

Suppose that $\beta(k)=r-1$, and consider minimal $a$ and maximal $b$ such that $a \leq k \leq b$ and $\beta(i)=r-1$ for $i \in[a, b]$. If there is no minimal $a$, we set $a=-\infty$. By Lemma 4.21, $\beta(a-1)=r-2$ and all the maps

$$
\begin{aligned}
\operatorname{HFL}^{-}\left(K_{r m, r n}, b, \ldots, b\right) & \stackrel{U_{1} \cdots U_{r}}{\longrightarrow} \operatorname{HFL}^{-}\left(K_{r m, r n}, b-1, \ldots, b-1\right) \rightarrow \ldots \\
\ldots \rightarrow & \rightarrow \operatorname{HFL}^{-}\left(K_{r m, r n}, a, \ldots, a\right) \stackrel{U_{1} \cdots U_{r}}{\longrightarrow} \operatorname{HFL}^{-}\left(K_{r m, r n}, a-1, \ldots, a-1\right)
\end{aligned}
$$

are surjective. Therefore

$$
R \cdot \operatorname{HFL}^{-}\left(K_{r m, r n}, b, \ldots, b\right) \simeq \mathcal{A}_{r}^{\mathrm{red}} /\left(U_{1} \cdots U_{r}\right)^{b-a} \mathcal{I}_{r-2}=: M_{r-1, b-a+1}
$$

is supported in all Alexander degrees with maximal coordinates in $[a, b]$ and in Alexander degrees with maximal coordinate $(a-1)$ which appears with multiplicity at least 2 .

Finally, we get the following decomposition of $\mathrm{HFL}^{-}$as an $R$-module:

$$
\operatorname{HFL}^{-}\left(K_{r m, r n}\right)=\bigoplus_{\substack{k: 0<\beta(k)<r-1 \\ \beta(k+1)<r-1}} M_{\beta(k)} \oplus \bigoplus_{\substack{a, b: \beta(a-1)=r-2 \\ \beta(b+1)<r-1 \\ \beta([a, b])=r-1}} M_{r-1, b-a+1} \oplus M_{r-1, \infty}
$$

Note that for $r=1$ we get $M_{0, l} \simeq \mathbb{F}\left[U_{1}\right] /\left(U_{1}^{l}\right)$ and $M_{0,+\infty} \simeq \mathbb{F}[U]$. 


\subsection{Spectral sequence for $\widehat{\mathrm{HFL}}$.}

Theorem 4.22. If $\beta(k)+\beta(k+1) \leq r-2$ then the spectral sequence for $\widehat{\mathrm{HFL}}\left(K_{r m, r n}, k, \ldots, k\right)$ degenerates at the $\widehat{E_{2}}$ page and

$$
\widehat{\operatorname{HFL}}\left(K_{r m, r n}, k, \ldots, k\right) \simeq \bigoplus_{i=0}^{\beta(k)}\left(\begin{array}{c}
r-1 \\
i
\end{array}\right) \mathbb{F}_{-2 \mathbf{h}(k)-i} \oplus \bigoplus_{i=0}^{\beta(k+1)}\left(\begin{array}{c}
r-1 \\
i
\end{array}\right) \mathbb{F}_{-2 \mathbf{h}(k)+2-r+i} .
$$

Proof. By Proposition 3.8, for a given $v$ there is a spectral sequence with $\widehat{E_{1}}$ page

$$
\widehat{E_{1}}=\bigoplus_{B \subset\{1, \ldots, r\}} \operatorname{HFL}^{-}\left(L, v+e_{B}\right)
$$

and converging to $\widehat{E}_{\infty}=\widehat{\mathrm{HFL}}(L, v)$. If $v=(k, \ldots, k)$ then (for $B \neq \emptyset$ ) the maximal coordinate of $v+e_{B}$ equals $k+1$ and appears with multiplicity $\lambda=|B|$. Therefore, by Theorem $5 \operatorname{HFL}^{-}\left(L, v+e_{B}\right)$ does not vanish if and only if either $B=\emptyset$ or $|B| \geq r-\beta(k+1)$, and it is given by Theorem 5 . By (1.1) we have $\mathbf{h}(k+1)=\mathbf{h}(k)-\beta(k+1)-1$.

The spectral sequence is bigraded by the homological (Maslov) grading at each vertex of the cube and the "cube grading" $|B|$. The differential $\widehat{\partial_{1}}$ acts along the edges of the cube, and decreases the Maslov grading by 2 and the cube grading by 1 .

One can check using Theorem 4.18 that its homology $\widehat{E_{2}}$ does not vanish in cube degrees 0 and $r-\beta(k+1)$, so one can write $\widehat{E_{2}}=\widehat{E_{2}^{0}} \oplus \widehat{E_{2}^{r-\beta(k+1)}}$, and

$$
\widehat{E_{2}^{0}} \simeq \bigoplus_{i=0}^{\beta(k)}\left(\begin{array}{c}
r-1 \\
i
\end{array}\right) \mathbb{F}_{-2 \mathbf{h}(k)-i}, \quad E_{2}^{r-\beta(k+1)} \simeq \bigoplus_{i=0}^{\beta(k+1)}\left(\begin{array}{c}
r-1 \\
i
\end{array}\right) \mathbb{F}_{-2 \mathbf{h}(k+1)-3 \beta(k+1)+i} .
$$

By (1.1) we have $\mathbf{h}(k+1)=\mathbf{h}(k)-\beta(k+1)-1$, so $-2 \mathbf{h}(k+1)-3 \beta(k+1)+i=-2 \mathbf{h}(k)+2-\beta(k+1)+i$.

A higher differential $\widehat{\partial}_{s}$ decreases the cube grading by $s$ and decreases the Maslov grading by $s+1$. Therefore the only nontrivial higher differential is $\widehat{\partial_{r-\beta(k+1)}}$ which vanishes by degree reasons too. Indeed, the maximal Maslov grading in $\widehat{E_{2}^{r-\beta(k+1)}}$ equals $-2 \mathbf{h}(k)+2$ while the minimal Maslov grading in $\widehat{E_{2}^{0}}$ equals $-2 \mathbf{h}(k)-\beta(k)$, so the differential can decrease the Maslov grading at most by $\beta(k)+2$. On the other hand, $\widehat{\partial_{r-\beta(k+1)}}$ drops it by $r-\beta(k+1)+1$, and for $\beta(k)+\beta(k+1)<r-1$ one has $r-\beta(k+1)+1>\beta(k)+2$. Therefore $\widehat{\partial_{r-\beta(k+1)}}=0$ and the spectral sequence vanishes at the $\widehat{E_{2}}$ page.

We illustrate the proof of Theorem 4.22 by Examples 5.4 and 5.5

Lemma 4.23. The following identity holds:

$$
\beta(1-k)+\beta(k)=r-2 .
$$

Proof. By (1.1) and Lemma 4.5 we have

$$
\beta(k)=h(k-1, \ldots, k-1)-h(k, \ldots, k)-1, \beta(1-k)=h(-k, \ldots,-k)-h(1-k, \ldots, 1-k)-1 .
$$

By Lemma 4.4 we have

$$
h(-k, \ldots,-k)=h(k, \ldots, k)+k r, h(1-k, \ldots, 1-k)=h(k-1, \ldots, k-1)+r(k-1) .
$$

These two identities imply the desired statement. 
Theorem 4.24. If $\beta(k)+\beta(k+1) \geq r-2$ then:

$$
\widehat{\operatorname{HFL}}\left(K_{r m, r n}, k, \ldots, k\right) \simeq \bigoplus_{i=0}^{r-2-\beta(k+1)}\left(\begin{array}{c}
r-1 \\
i
\end{array}\right) \mathbb{F}_{-2 \mathbf{h}(k)-i} \oplus \bigoplus_{i=0}^{r-2-\beta(k)}\left(\begin{array}{c}
r-1 \\
i
\end{array}\right) \mathbb{F}_{-2 \mathbf{h}(k)+2-r+i}
$$

Proof. By Lemma 4.23 we get $\beta(-k)=r-2-\beta(k+1)$ and $\beta(1-k)=r-2-\beta(k)$, so

$$
\beta(k)+\beta(k+1)+\beta(-k)+\beta(1-k)=2(r-2),
$$

so $\beta(-k)+\beta(1-k) \leq r-2$. By Theorem 4.22 the spectral sequence degenerates for $\widehat{\operatorname{HFL}}(-k, \ldots,-k)$ and

$$
\widehat{\operatorname{HFL}}\left(K_{r m, r n},-k, \ldots,-k\right) \simeq \bigoplus_{i=0}^{r-2-\beta(k+1)}\left(\begin{array}{c}
r-1 \\
i
\end{array}\right) \mathbb{F}_{-2 \mathbf{h}(-k)-i} \oplus \bigoplus_{i=0}^{r-2-\beta(k)}\left(\begin{array}{c}
r-1 \\
i
\end{array}\right) \mathbb{F}_{-2 \mathbf{h}(-k)+2-r+i}
$$

Finally, by [OS08, Proposition 8.2] we have

$$
\widehat{\mathrm{HFL}} \bullet\left(K_{r m, r n}, k, \ldots, k\right)=\widehat{\mathrm{HFL}} \bullet-2 k r\left(K_{r m, r n},-k, \ldots,-k\right)
$$

and by Lemma $4.4 \mathbf{h}(k)=\mathbf{h}(-k)-k r$.

Theorem 4.25. Off-diagonal homology groups are supported on the union of the unit cubes along the diagonal. In such a cube with corners $(k, \ldots, k)$ and $(k+1, \ldots, k+1)$ one has

$$
\widehat{\operatorname{HFL}}\left(K_{r m, r n},(k-1)^{j}, k^{r-j}\right) \simeq\left(\begin{array}{c}
r-2 \\
\beta(k)
\end{array}\right) \mathbb{F}_{-2 \mathbf{h}(k)-\beta(k)-j} .
$$

Proof. We use the spectral sequence from $\mathrm{HFL}^{-}$to $\widehat{\mathrm{HFL}}$. By Theorem 4.18, all the $\widehat{E_{2}}$ homology outside the union of these cubes vanish (since some $U_{i}$ would provide an isomorphism between $\operatorname{HFL}^{-}\left(K_{r m, r n}, v\right)$ and $\left.\operatorname{HFL}^{-}\left(K_{r m, r n}, v-e_{i}\right)\right)$. Furthermore, if $\beta(k)=r-1$ then the homology in the cube vanish too, so we can focus on the case $\beta(k) \leq r-2$.

One can check that $\widehat{E_{2}}$ does not vanish in cube degrees $j-\beta(k), \ldots, j$ and

$$
\widehat{E_{2}^{j-c}} \simeq\left(\begin{array}{c}
j-1 \\
c
\end{array}\right)\left(\begin{array}{c}
r-1-j \\
\beta(k)-c
\end{array}\right) \mathbb{F}_{-2 \mathbf{h}(k)-\beta(k)-c} .
$$

Note that the total homological degree on $\widehat{E_{2}^{j-c}}$ equals $-2 \mathbf{h}(k)-\beta(k)-j$ and does not depend on $c$. Therefore all higher differentials in the spectral sequence must vanish and the rank of $\widehat{\mathrm{HFL}}$ equals:

$$
\sum_{c=0}^{\beta}\left(\begin{array}{c}
j-1 \\
c
\end{array}\right)\left(\begin{array}{c}
r-1-j \\
\beta(k)-c
\end{array}\right)=\left(\begin{array}{c}
r-2 \\
\beta(k)
\end{array}\right) \text {. }
$$

We illustrate this proof by Example 5.6.

4.5. Special case: $m=1, n=2 g(K)-1$. The case $m=1, n=2 g(K)-1$ is special since Lemma 4.6 is not always true. Indeed, $K_{m, n}=K$ and $l=n=2 g(K)-1$, but for $v=g(K)-l=1-g(K)$ we have $\operatorname{HFL}^{-}(K, v)=0$. However, it is clear that in all other cases Lemma 4.6 is true, so for generic $v$ Lemmas 4.8 and 4.10 hold true. This allows one to prove an analogue of Theorem 5 .

Theorem 4.26. Assume that $m=1, n=2 g(K)-1$ (so $l=2 g(K)-1$ ) and suppose that $v=$ $\left(u_{1}^{\lambda_{1}}, u_{2}^{\lambda_{2}}, \ldots, u_{s}^{\lambda_{s}}\right)$ where $u_{1}<\ldots<u_{s}$. Then the Heegaard-Floer homology group $\operatorname{HFL}^{-}\left(K_{r m, r n}, v\right)$ can be described as following: 
(a) Assume that $u_{s}-c+l\left(r-\lambda_{s}\right)=g(K)-\nu l$ with $1 \leq \nu \leq \lambda_{s}$. Then

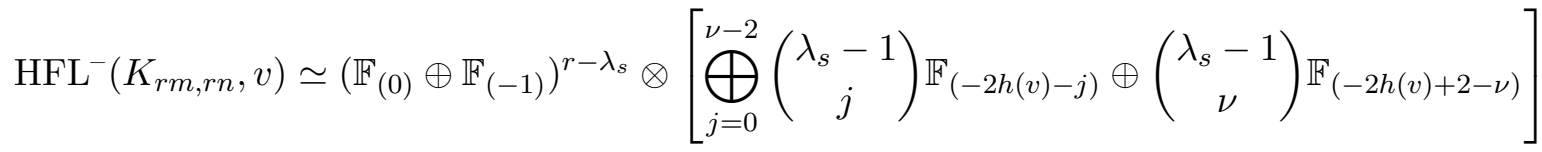

(b) In all other cases, the homology is given by Theorem 5.

Proof. One can check that the proof of Lemma 4.8 fails if $u_{s}-c+l\left(r-\lambda_{s}\right)=g(K)-l$, and remains true in all other cases. Similarly, the proof of Lemma 4.10 fails only if $u_{s}-c+l\left(r-\lambda_{s}\right)+l j=g(K)-l$ for $1 \leq j \leq \lambda_{s}-1$, which is equivalent to $u_{s}-c+l\left(r-\lambda_{s}\right)=g(K)-(j+1)$. This proves (b).

Let us consider the special case (a). Note that

$$
\begin{gathered}
h_{m, n}\left(u_{s}-c+l\left(r-\lambda_{s}\right)+l j-1\right)-h_{m, n}\left(u_{s}-c+l\left(r-\lambda_{s}\right)+l j\right)= \\
\chi\left(\operatorname{HFK}^{-}(K, g(K)+l(j-\nu))= \begin{cases}1, & \text { if } j<\nu-1 \\
0, & \text { if } j=\nu-1 \\
1, & \text { if } j=\nu \\
0, & \text { if } j>\nu .\end{cases} \right.
\end{gathered}
$$

Given a pair of subsets $B^{\prime} \subset\left\{1, \ldots, r-\lambda_{s}\right\}$ and $B^{\prime \prime} \subset\left\{r-\lambda_{s}+1, \ldots, r\right\}$, one can write, analogously to Lemma 4.10:

$$
h_{r m, r n}\left(v-e_{B^{\prime}}-e_{B^{\prime \prime}}\right)=h_{r m, r n}(v)+\left|B^{\prime}\right|+w\left(B^{\prime \prime}\right),
$$

where

$$
w\left(B^{\prime \prime}\right)= \begin{cases}\left|B^{\prime \prime}\right|, & \text { if }\left|B^{\prime \prime}\right| \leq \nu-1 \\ \nu-1, & \text { if }\left|B^{\prime \prime}\right|=\nu \\ \nu, & \text { if }\left|B^{\prime \prime}\right|>\nu\end{cases}
$$

By the Künneth formula, the $E_{2}$ page of the spectral sequence is determined by the "deformed cube homology" with the weight function $w\left(B^{\prime \prime}\right)$, as in (4.5). If $\partial$, as above, denotes the standard cube differential, then, similarly to Lemma 4.14, the homology of $\partial_{U}^{w}$ is isomorphic to the kernel of $\partial$ in cube degrees $0, \ldots \nu-2$ and $\nu$.

Finally, we need to prove that all higher differentials vanish. For a homology generator $\alpha$ on the $E_{2}$ page of cube degree $x$, its bidegree is equal either to $(x,-2 h(v)-2 x)$ or to $(x,-2 h(v)-2 x+2)$. The differential $\partial_{k}$ has bidegree $(-k, k-1)$ (see Remark 3.6), so the bidegree of $\partial_{k}(\alpha)$ is equal either to $(x-k,-2 h(v)-2 x+k-1)$ or to $(x-k,-2 h(v)-2 x+k+1)$. Since $-2 x+k+1<-2(x-k)$ for $k>1$, we have $\partial_{k}(\alpha)=0$.

The action of $U_{i}$ in this special case can be described similarly to Theorem 4.18. However, it is not true that $U_{i}$ is surjective whenever it does not obviously vanish. In particular, the following example shows that $\mathrm{HFL}^{-}$may be not generated by diagonal classes, so Theorem 6 does not hold. We leave the appropriate adjustment of Theorem 6 as an exercise to a reader.

Example 4.27. Consider $T_{2,2}$, the $(2,2)$ cable of the trefoil. We have $g(K)=l=1$ and $c=1 / 2$, so by Theorem 4.26

$$
\operatorname{HFL}^{-}\left(T_{2,2}, 1 / 2,1 / 2\right) \simeq \mathbb{F}_{(-1)}, \quad \operatorname{HFL}^{-}\left(T_{2,2},-1 / 2,1 / 2\right) \simeq \mathbb{F}_{(-2)} \oplus \mathbb{F}_{(-3)} .
$$

Therefore $U_{1}$ is not surjective. Furthermore, the class in $\operatorname{HFL}^{-}\left(T_{2,2},-1 / 2,1 / 2\right)$ of homological degree $(-2)$ is not in the image of any diagonal class under the $R$-action. 


\section{EXAMPLES}

5.1. $(n, n)$ torus links. The symmetrized multi-variable Alexander polynomial of the $(n, n)$ torus link equals (for $n>1$ ):

$$
\Delta_{T_{n, n}}\left(t_{1}, \ldots, t_{n}\right)=\left(\left(t_{1} \cdots t_{n}\right)^{1 / 2}-\left(t_{1} \cdots t_{n}\right)^{-1 / 2}\right)^{n-2} .
$$

Each pair of components has linking number 1 , so $c=(n-1) / 2$. The homology groups $\mathrm{HFL}^{-}(T(n, n), v)$ are described by the following theorem, which is a special case of Theorem 5 .

Theorem 5.1. Consider the $(n, n)$ torus link, and an Alexander grading $v=\left(v_{1}, \ldots, v_{n}\right)$. Suppose that among the coordinates $v_{i}$ exactly $\lambda$ are equal to $k$ and all other coordinates are less than $k$. Let $|v|=v_{1}+\ldots+v_{n}$. Then

$$
\operatorname{HFL}^{-}(T(n, n), v)= \begin{cases}0 & \text { if } k>\lambda-\frac{n+1}{2}, \\
\left(\mathbb{F}_{(0)} \oplus \mathbb{F}_{(-1)}\right)^{n-1} \otimes \mathbb{F}_{2|v|} & \text { if } k<-\frac{n-1}{2}, \\
\left(\mathbb{F}_{(0)} \oplus \mathbb{F}_{(-1)}\right)^{n-\lambda} \otimes \bigoplus_{i=0}^{\lambda-\frac{n+1}{2}-k}\left(\begin{array}{c}
\lambda-1 \\
i
\end{array}\right) \mathbb{F}_{(-2 h(v)-i)} & \text { if }-\frac{n-1}{2} \leq k \leq \lambda-\frac{n+1}{2},\end{cases}
$$

where $h(v)=\frac{1}{2}\left(\frac{n-1}{2}-k\right)\left(\frac{n-1}{2}-k+1\right)+k n-|v|$ in the last case.

Proof. Indeed, $\beta(k)=\frac{n-1}{2}-k$ for $k>-\frac{n-1}{2}$ and $\beta(k)=n-1$ for $k \leq-\frac{n-1}{2}$. By Theorem 5 , the homology group $\mathrm{HFL}^{-}(T(n, n), v)$ does not vanish if and only if

$$
k \leq \lambda-\frac{n+1}{2} .
$$

If $k \geq-\frac{n-1}{2}$, equation (4.3) implies:

$$
h_{n, n}(v)=\frac{1}{2}\left(\frac{n-1}{2}-k\right)\left(\frac{n-1}{2}-k+1\right)+k n-|v| .
$$

If $k \leq-\frac{n-1}{2}$, equation (4.3) implies $h_{n, n}(v)=-|v|$. Furthermore, for all $v$ satisfying (5.1) one has

$$
\operatorname{HFL}^{-}(T(n, n), v)=\left(\mathbb{F}_{(0)} \oplus \mathbb{F}_{(-1)}\right)^{n-\lambda} \otimes \bigoplus_{j=0}^{\lambda-\frac{n+1}{2}-k}\left(\begin{array}{c}
\lambda-1 \\
j
\end{array}\right) \mathbb{F}_{\left(-2 h_{n, n}(v)-j\right)}
$$

Finally, if $k-\frac{n-1}{2}$, then (5.1) holds for all $\lambda$ and $\lambda-\frac{n+1}{2}-k>\lambda-1$, hence

$\operatorname{HFL}^{-}(T(n, n), v)=\left(\mathbb{F}_{(0)} \oplus \mathbb{F}_{(-1)}\right)^{n-\lambda} \otimes \bigoplus_{j=0}^{\lambda-1}\left(\begin{array}{c}\lambda-1 \\ j\end{array}\right) \mathbb{F}_{\left(-2 h_{n, n}(v)-j\right)}=\left(\mathbb{F}_{(0)} \oplus \mathbb{F}_{(-1)}\right)^{n-1} \otimes \mathbb{F}_{\left(-2 h_{n, n}(v)\right)}$

Remark 5.2. One can check that, in agreement with [GN15], the condition (5.1) defines the multidimensional semigroup of the plane curve singularity $x^{n}=y^{n}$.

Corollary 5.3. We have the following decomposition of $\mathrm{HFL}^{-}$as an $R$-module:

$$
\mathrm{HFL}^{-}(T(n, n))=M_{0} \oplus M_{1} \oplus M_{2} \oplus \ldots \oplus M_{n-2} \oplus M_{n-1,+\infty} .
$$

To prove Theorem 4, we use Theorem 3.

Proof of Theorem 4. We have $\beta\left(\frac{n-1}{2}-s\right)=s$ for $s<n-1$, and

$$
\beta\left(\frac{n-1}{2}-s\right)+\beta\left(\frac{n-1}{2}-s+1\right)=2 s-1 \leq n-2 \leq s \leq \frac{n-1}{2} .
$$


Therefore for $s \leq \frac{n-1}{2}$ Theorem 4.22 implies the degeneration of the spectral sequence from $\mathrm{HFL}^{-}$ to $\widehat{\mathrm{HFL}}$, and

$$
\widehat{\operatorname{HFL}}\left(T(n, n), \frac{n-1}{2}-s, \ldots, \frac{n-1}{2}-s\right)=\bigoplus_{i=0}^{s}\left(\begin{array}{c}
n-1 \\
i
\end{array}\right) \mathbb{F}_{\left(-s^{2}-s-i\right)} \oplus \bigoplus_{i=0}^{s-1}\left(\begin{array}{c}
n-1 \\
i
\end{array}\right) \mathbb{F}_{\left(-s^{2}-s-n+2+i\right)} .
$$

Let us illustrate the degeneration of the spectral sequence from $\mathrm{HFL}^{-}$to $\widehat{\mathrm{HFL}}$ in some examples.

Example 5.4. For $s=0$ we have $\widehat{E_{1}}=\widehat{E_{2}}=\mathbb{F}_{(0)}$. For $s=1$ the $\widehat{E_{1}}$ page has nonzero entries in cube degree 0 where one gets

$$
\operatorname{HFL}^{-}\left(T(n, n), \frac{n-1}{2}-1, \ldots, \frac{n-1}{2}-1\right) \simeq \mathbb{F}_{(-2)} \oplus(n-1) \mathbb{F}_{(-3)},
$$

and in cube degree $n$ where one gets $\mathbb{F}_{(0)}$. Indeed, the differential $\widehat{\partial_{1}}$ vanishes, so for $n>2$

$$
\widehat{\mathrm{HFL}}\left(T(n, n), \frac{n-1}{2}-1, \ldots, \frac{n-1}{2}-1\right) \simeq \mathbb{F}_{(-2)} \oplus(n-1) \mathbb{F}_{(-3)} \oplus \mathbb{F}_{(-n)} .
$$

Note that for $n=2$ the differential $\widehat{\partial_{2}}$ does not vanish, so the bound $s \leq \frac{n-1}{2}$ is indeed necessary for the spectral sequence to collapse at $\widehat{E_{2}}$ page.

Example 5.5. The case $s=2$ is more interesting. The $\widehat{E_{1}}$ page has nonzero entries in cube degree $0, n-1$ (where we have $n$ vertices) and $n$, where one has $\widehat{E_{1}^{0}}=\mathbb{F}_{(-6)} \oplus(n-1) \mathbb{F}_{(-7)} \oplus\left(\begin{array}{c}n-1 \\ 2\end{array}\right) \mathbb{F}_{(-8)}, \widehat{E_{1}^{n-1}}=n\left(\mathbb{F}_{(-4)} \oplus \mathbb{F}_{(-5)}\right), \widehat{E_{1}^{n}}=\mathbb{F}_{(-2)} \oplus(n-1) \mathbb{F}_{(-3)}$

The differential $\widehat{\partial_{1}}$ cancels some summands in $\widehat{E_{1}^{n-1}}$ and $\widehat{E_{1}^{n}}$;

$$
\widehat{E_{2}^{0}}=\mathbb{F}_{(-6)} \oplus(n-1) \mathbb{F}_{(-7)} \oplus\left(\begin{array}{c}
n-1 \\
2
\end{array}\right) \mathbb{F}_{(-8)}, \widehat{E_{2}^{n-1}}=(n-1) \mathbb{F}_{(-4)}+\mathbb{F}_{(-5)} .
$$

For $n>4$ all higher differentials vanish and

$$
\begin{aligned}
\widehat{\mathrm{HFL}}\left(T(n, n), \frac{n-1}{2}-2, \ldots, \frac{n-1}{2}-2\right) \simeq \\
\quad \mathbb{F}_{(-6)} \oplus(n-1) \mathbb{F}_{(-7)} \oplus\left(\begin{array}{c}
n-1 \\
2
\end{array}\right) \mathbb{F}_{(-8)} \oplus(n-1) \mathbb{F}_{(-3-n)}+\mathbb{F}_{(-4-n)} .
\end{aligned}
$$

The following example illustrates the computation of $\widehat{\mathrm{HFL}}$ for the off-diagonal Alexander gradings.

Example 5.6. Let us compute the homology $\widehat{\operatorname{HFL}}(T(n, n), v)$ for $v=\left(\frac{n-1}{2}-2\right)^{j}\left(\frac{n-1}{2}-1\right)^{n-j}(1 \leq$ $j \leq n-1)$ using the spectral sequence from $\mathrm{HFL}^{-}$. In the $n$ dimensional cube $\left(v+e_{B}\right)$ almost all all vertices have vanishing $\mathrm{HFL}^{-}$, except for the vertex $\left(\frac{n-1}{2}-1, \ldots, \frac{n-1}{2}-1\right)$

$$
\operatorname{HFL}^{-}\left(\frac{n-1}{2}-1, \ldots, \frac{n-1}{2}-1\right)=F_{(-2)} \oplus(n-1) \mathbb{F}_{(-3)}
$$

and $j$ of its neighbors with homology $\mathbb{F}_{(-4)} \oplus \mathbb{F}_{(-5)}$. Clearly, $\widehat{E_{2}}$ is concentrated in degrees $j$ (with homology $\left.(n-1-j) \mathbb{F}_{(-3)}\right)$ and $(j-1)$ (with homology $\left.(j-1) \mathbb{F}_{(-4)}\right)$. Note that both parts contribute to the total degree $(-3-j)$, so

$$
\widehat{\operatorname{HFL}}(T(n, n), v)=(n-1-j) \mathbb{F}_{(-3-j)} \oplus(j-1) \mathbb{F}_{(-3-j)}=(n-2) \mathbb{F}_{(-3-j)} .
$$


Finally, we draw all the homology groups $\mathrm{HFL}^{-}$for $(2,2)$ and $(3,3)$ torus links.

Example 5.7. For the Hopf link, one has two cases. If $v_{1}<v_{2}$, then the condition (5.1) implies $v_{2} \leq-1 / 2$. If $v_{1}=v_{2}$, then (5.1) implies $v_{2} \geq 1 / 2$.

The nonzero homology of the Hopf link is shown in Figure 3 and Table 1

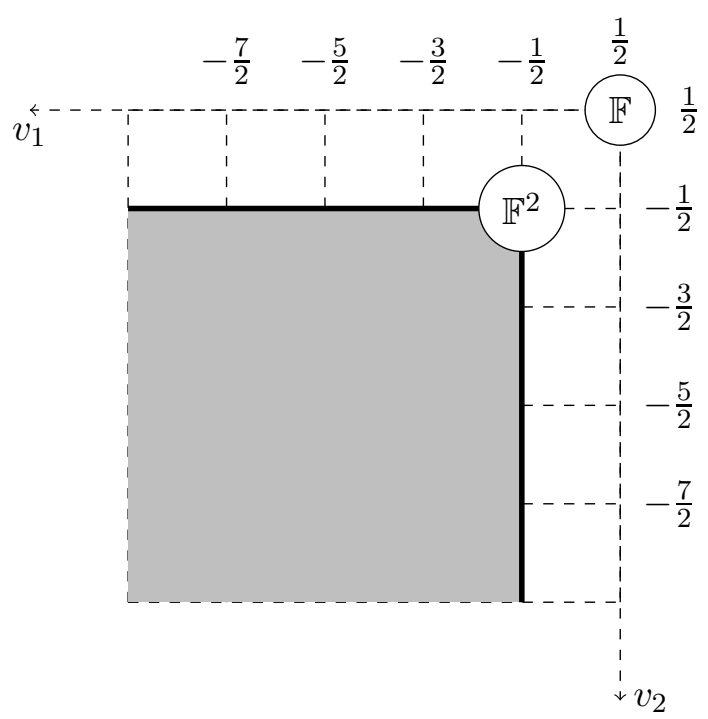

Figure 3 . HFL ${ }^{-}$for the $(2,2)$ torus link: $\mathbb{F}^{2}$ on thick lines and in the grey region

\begin{tabular}{|c|c|}
\hline Alexander grading & Homology \\
\hline$(1 / 2,1 / 2)$ & $\mathbb{F}_{(0)}$ \\
\hline$(a, b), a, b \leq-1 / 2$ & $\mathbb{F}_{(2 a+2 b)} \oplus \mathbb{F}_{(2 a+2 b-1)}$ \\
\hline
\end{tabular}

TABLE 1. Maslov gradings for the $(2,2)$ torus link

Example 5.8. For the $(3,3)$ torus link, one has two cases. If $v_{1} \leq v_{2}<v_{3}$, then the condition (5.1) implies $v_{3} \leq 1$. If $v_{1}<v_{2}=v_{3}$, then (5.1) implies $v_{3} \leq 0$. Finally, if $v_{1}=v_{2}=v_{3}$, then (5.1) implies $v_{3} \leq 1$. In other words, nonzero homology appears at the point $(1,1,1)$, at three lines $(0,0, k),(0, k, 0),(k, 0,0)(k \leq 0)$ and at the octant $\max \left(v_{1}, v_{2}, v_{3}\right) \leq-1$.

This homology is shown in Figure 4 and Table 2.

\begin{tabular}{|c|c|}
\hline Alexander grading & Homology \\
\hline$(1,1,1)$ & $\mathbb{F}_{(0)}$ \\
\hline$(0,0,0)$ & $\mathbb{F}_{(-2)} \oplus 2 \mathbb{F}_{(-3)}$ \\
\hline$(0,0, k),(0, k, 0)$ and $(k, 0,0)(k<0)$ & $\mathbb{F}_{(2 k-2)} \oplus \mathbb{F}_{(2 k-3)}$ \\
\hline$(a, b, c), a, b, c \leq-1$ & $\mathbb{F}_{(2 a+2 b+2 c)} \oplus 2 \mathbb{F}_{(2 a+2 b+2 c-1)} \oplus \mathbb{F}_{(2 a+2 b+2 c-2)}$ \\
\hline
\end{tabular}

TABLE 2. Maslov gradings for the $(3,3)$ torus link 


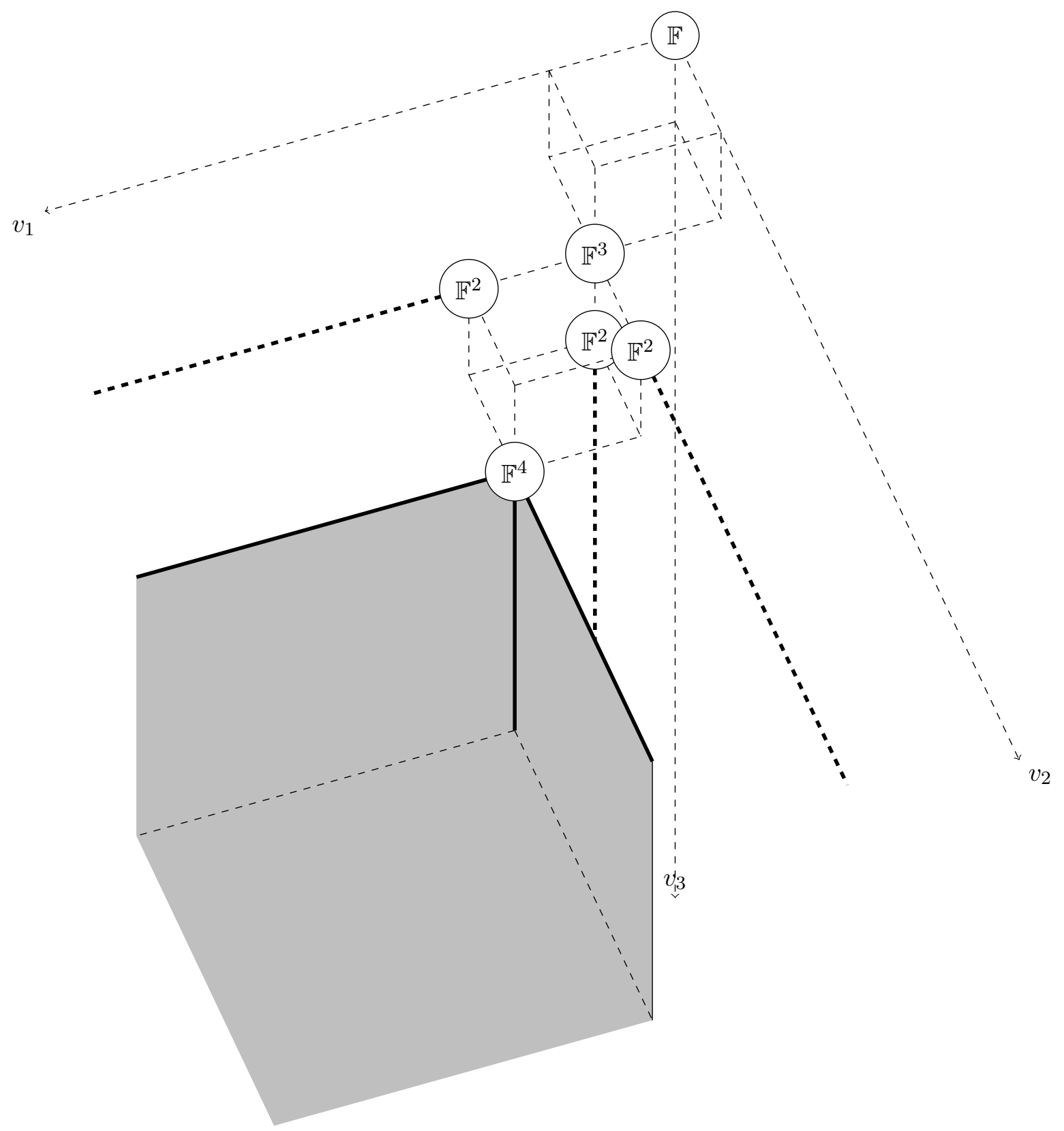

Figure 4 . HFL ${ }^{-}$for the $(3,3)$ torus link: $\mathbb{F}^{2}$ on dashed thick lines; $\mathbb{F}^{4}$ on solid thick lines and in the shaded region. Top Alexander grading is $(1,1,1)$.

5.2. More general torus links. The $\mathrm{HFL}^{-}$homology of the $(4,6)$ torus link is shown in Figure 5 and Table 3 . Note that as an $\mathbb{F}\left[U_{1}, U_{2}\right]$ module it can be decomposed into 5 copies of $M_{0} \simeq \mathbb{F}$, a copy of $M_{1,1}$ and a copy of $M_{1,+\infty}$. In particular, the map $U_{1} U_{2}: \mathrm{HFL}^{-}(-2,-2) \rightarrow \mathrm{HFL}^{-}(-3,-3)$ is surjective with one-dimensional kernel.

5.3. Non-algebraic example. In this subsection we compute the Heegaard-Floer homology for the $(4,6)$-cable of the trefoil. Its components are $(2,3)$-cables of the trefoil, which are known to be 


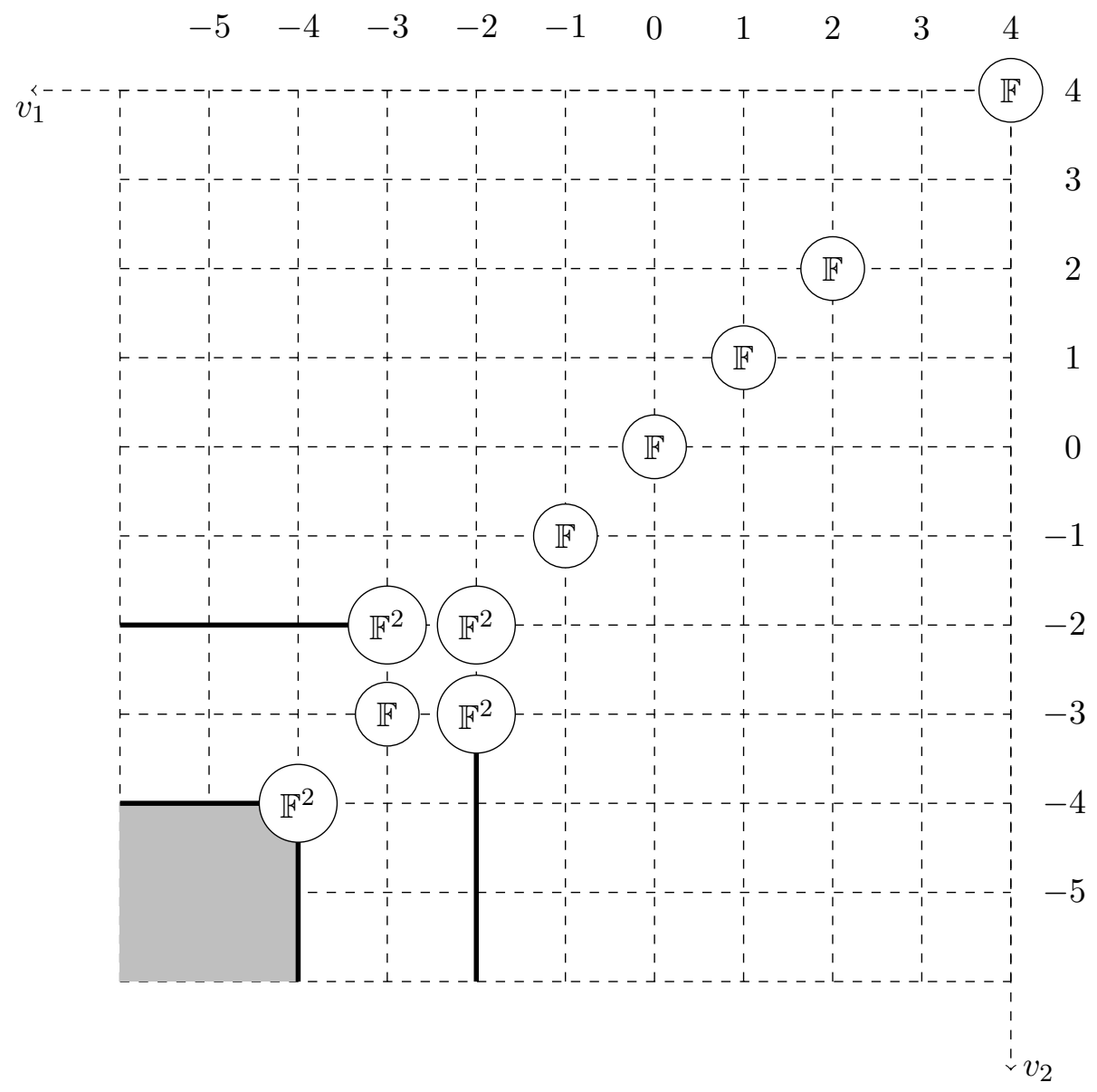

Figure 5. $\mathrm{HFL}^{-}$for the $(4,6)$ torus link: $\mathbb{F}^{2}$ on thick lines and in the grey region

\begin{tabular}{|c|c|}
\hline Alexander grading & Homology \\
\hline$(4,4)$ & $\mathbb{F}_{(0)}$ \\
\hline$(2,2)$ & $\mathbb{F}_{(-2)}$ \\
\hline$(1,1)$ & $\mathbb{F}_{(-4)}$ \\
\hline$(0,0)$ & $\mathbb{F}_{(-6)}$ \\
\hline$(-1,-1)$ & $\mathbb{F}_{(-8)}$ \\
\hline$(-2, k)$ and $(k,-2), k \leq-2$ & $\mathbb{F}_{(2 k-6)} \oplus \mathbb{F}_{(2 k-7)}$ \\
\hline$(-3,-3)$ & $\mathbb{F}_{(-12)}$ \\
\hline$(a, b), a, b \leq-4$ & $\mathbb{F}_{(2 a+2 b)} \oplus \mathbb{F}_{(2 a+2 b-1)}$ \\
\hline
\end{tabular}

TABLE 3. Maslov gradings for the $(4,6)$ torus link

L-space knots (cf. [Hed09]), but not algebraic knots. By Theorem 2, the (4,6)-cable of the trefoil is an L-space link, but its homology is not covered by [GN15]. 
The Alexander polynomial of the $(2,3)$-cable of the trefoil equals:

$$
\Delta_{T_{2,3}}(t)=\frac{\left(t^{6}-t^{-6}\right)\left(t^{1 / 2}-t^{-1 / 2}\right)}{\left(t^{3 / 2}-t^{-3 / 2}\right)\left(t^{2}-t^{-2}\right)}
$$

hence the Euler characteristic of its Heegaard-Floer homology equals

$$
\chi_{2,3}(t)=\frac{\Delta_{T_{2,3}}(t)}{1-t^{-1}}=t^{3}+1+t^{-1}+t^{-3}+t^{-4}+\ldots
$$

By (4.1), the bivariate Alexander polynomial of the $(4,6)$-cable equals:

$$
\begin{gathered}
\chi_{4,6}\left(t_{1}, t_{2}\right)=\chi_{2,3}\left(t_{1} \cdot t_{2}\right)\left(\left(t_{1} t_{2}\right)^{3}-\left(t_{1} t_{2}\right)^{-3}\right) \\
=\left(t_{1} t_{2}\right)^{6}+\left(t_{1} t_{2}\right)^{3}+\left(t_{1} t_{2}\right)^{2}+\left(t_{1} t_{2}\right)^{-1}+\left(t_{1} t_{2}\right)^{-2}+\left(t_{1} t_{2}\right)^{-5} .
\end{gathered}
$$

The nonzero Heegaard-Floer homology are shown in Figure 6 and the corresponding Maslov gradings are given in Table 4 . Note that as $\mathbb{F}\left[U_{1}, U_{2}\right]$ module it can be decomposed in the following way:

$$
\mathrm{HFL}^{-} \simeq 4 M_{0} \oplus M_{1,1} \oplus M_{1,2} \oplus M_{1,+\infty}
$$

\section{REFERENCES}

[GN15] Eugene Gorsky and András Némethi, Lattice and Heegaard-Floer homologies of algebraic links, Int. Math. Res. Not. IMRN (2015).

[Hed09] Matthew Hedden, On knot Floer homology and cabling II, Int. Math. Res. Not. IMRN (2009), no. 12, $2248-2274$.

[Hei74] Wolfgang Heil, Elementary surgery on Seifert fiber spaces, Yokohama Math. J. 22 (1974), 135-139.

[HLZ13] Jennifer Hom, Tye Lidman, and Nicholas Zufelt, Reducible surgeries and Heegaard Floer homology, 2013, Preprint, arXiv:1307.5317.

[Hom11] Jennifer Hom, A note on cabling and L-space surgeries, Algebr. Geom. Topol. 11 (2011), no. 1, $219-223$.

[HW15] Jonathan Hanselman and Liam Watson, A calculus for bordered Floer homology, 2015, Preprint, arXiv:1508.05445.

[Lic12] Joan Licata, Heegaard Floer homology of $(n, n)$-torus links: computations and questions, 2012, Preprint, arXiv:1208.0394.

[Liu14] Yajing Liu, L-space surgeries on links, 2014, Preprint, arXiv:1409.0075v1.

[LOT08] Robert Lipshitz, Peter Ozsváth, and Dylan Thurston, Bordered Heegaard Floer homology: Invariance and pairing, 2008, Preprint, arXiv:0810.0687.

[LS07] Paolo Lisca and András I. Stipsicz, Ozsváth-Szabó invariants and tight contact 3-manifolds. III, J. Symplectic Geom. 5 (2007), no. 4, 357-384.

[MO10] Ciprian Manolescu and Peter Ozsváth, Heegaard Floer homology and integer surgeries on links, 2010, Preprint, arXiv:1011.1317v1.

[OS04a] Peter Ozsváth and Zoltán Szabó, Holomorphic disks and three-manifold invariants: properties and applications, Ann. of Math. (2) 159 (2004), no. 3, 1159-1245.

[OS04b] _ Holomorphic disks and topological invariants for closed three-manifolds, Ann. of Math. (2) 159 (2004), no. 3, 1027-1158.

[OS05] - On knot Floer homology and lens space surgeries, Topology 44 (2005), no. 6, 1281-1300.

[OS08] Holomorphic disks, link invariants and the multi-variable Alexander polynomial., Algebr. Geom. Topol. 8 (2008), no. 2, 615-692.

[OS11] Knot Floer homology and rational surgeries, Algebr. Geom. Topol. 11 (2011), no. 1, 1-68.

[Shi85] Tetsuo Shibuya, On the genus of torus links, Kobe J. Math. 2 (1985), no. 2, 123-125.

Department of Mathematics, Columbia University, 2990 Broadway, New York, NY 10027

Department of Mathematics, UC Davis, One Shields Ave, Davis, CA 95616 


\begin{tabular}{|c|c|}
\hline Alexander grading & Homology \\
\hline$(6,6)$ & $\mathbb{F}_{(0)}$ \\
\hline$(3,3)$ & $\mathbb{F}_{(-2)}$ \\
\hline$(2,2)$ & $\mathbb{F}_{(-4)}$ \\
\hline$(0, k)$ and $(k, 0), k \geq 0$ & $\mathbb{F}_{(2 k-6)} \oplus \mathbb{F}_{(2 k-7)}$ \\
\hline$(-1,-1)$ & $\mathbb{F}_{(-10)}$ \\
\hline$(-2,-2)$ & $\mathbb{F}_{(-12)}$ \\
\hline$(-3, k)$ and $(k,-3), k \geq-3$ & $\mathbb{F}_{(2 k-8)} \oplus \mathbb{F}_{(2 k-9)}$ \\
\hline$(-4, k)$ and $(k,-4), k \geq 10$ & $\mathbb{F}_{(2 k-10)} \oplus \mathbb{F}_{(2 k-11)}$ \\
\hline$(-5,-5)$ & $\mathbb{F}_{(-22)}$ \\
\hline$(a, b), a, b \leq-6$ & $\mathbb{F}_{(2 a+2 b)} \oplus \mathbb{F}_{(2 a+2 b-1)}$ \\
\hline
\end{tabular}

TABLE 4. Maslov gradings for the $(4,6)$ cable of the trefoil

International Laboratory of Representation Theory and Mathematical Physics, NRU-HSE, 7 Vavilova St., Moscow, Russia 117312

E-mail address: egorsky@math.columbia.edu

Department of Mathematics, Columbia University, 2990 Broadway, New York, NY 10027

E-mail address: hom@math.columbia.edu 


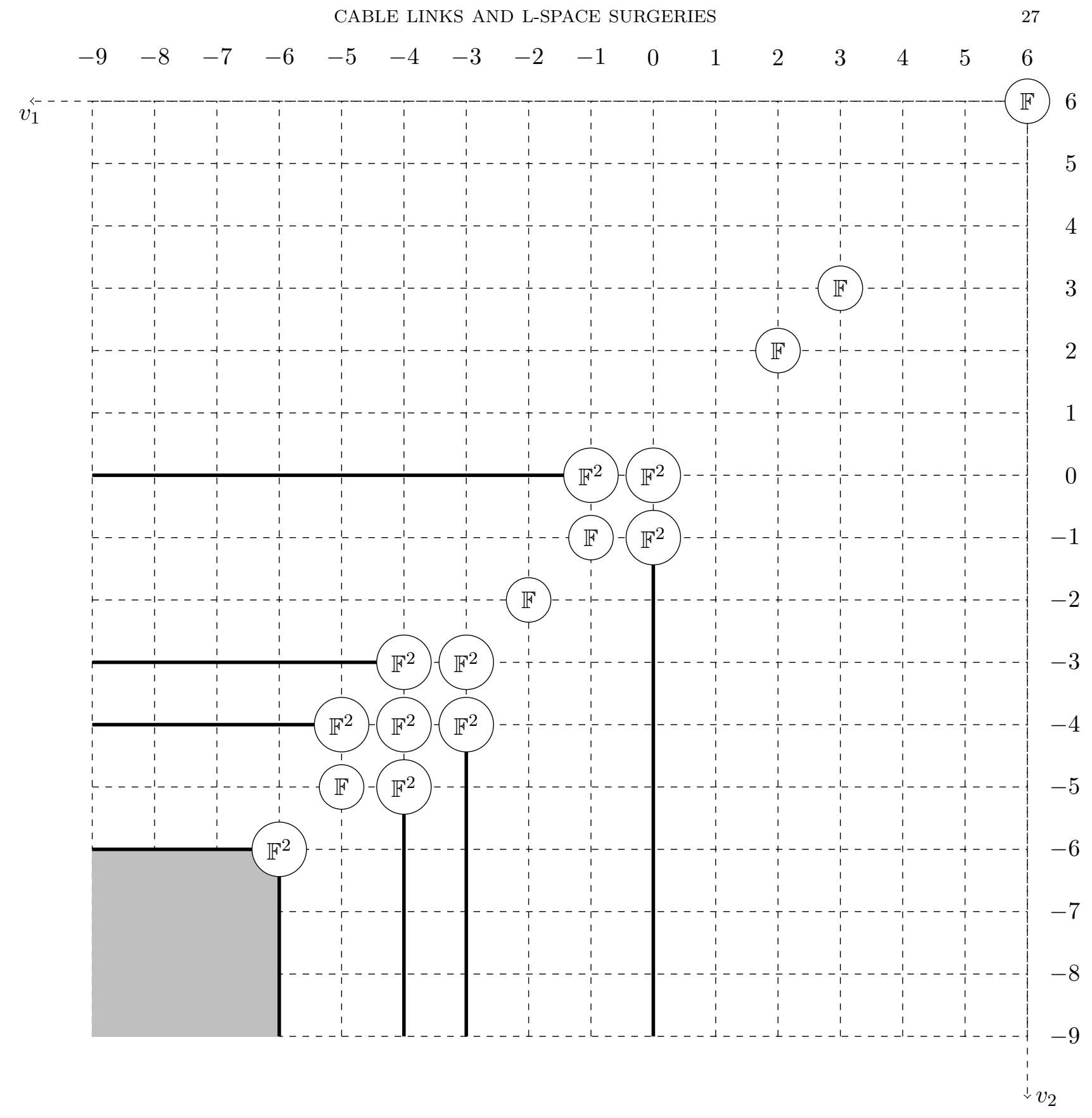

Figure 6 . HFL ${ }^{-}$for the $(4,6)$ cable of the trefoil: $\mathbb{F}^{2}$ on thick lines and in the grey region 